\title{
The KMS Condition for the homoclinic equivalence relation and Gibbs probabilities
}

\author{
A. O. Lopes and G. Mantovani
}

March 29, 2019

\begin{abstract}
D. Ruelle considered a general setting where he is able to characterize equilibrium states for Hölder potentials based on properties of conjugating homeomorphism in the so called Smale spaces. On this setting he also shows a relation of KMS states of $C^{*}$-algebras with equilibrium probabilities of Thermodynamic Formalism. A later paper by N. Haydn and D. Ruelle presents a shorter proof of this equivalence.

Here we consider similar problems but now on the symbolic space $\Omega=\{1,2, \ldots, d\}^{\mathbb{Z}-\{0\}}$ and the dynamics will be given by the shift $\tau$. In the case of potentials depending on a finite coordinates we will present a simplified proof of the equivalence mentioned above which is the main issue of the papers by D. Ruelle and N. Haydn. The class of conjugating homeomorphism is explicit and reduced to a minimal set of conditions.

We also present with details (following D. Ruelle) the relation of these probabilities with the KMS dynamical $C^{*}$-state on the $C^{*}$ Algebra associated to the groupoid defined by the homoclinic equivalence relation.

The topics presented here are not new but we believe the main ideas of the proof of the results by Ruelle and Haydn will be quite transparent in our exposition.
\end{abstract}

\section{Introduction}

D. Ruelle in 20] considered a general setting (which includes hyperbolic diffeomorphisms on manifolds) where he is able to describe a formulation of the concept of Gibbs state based on conjugating homeomorphism in the 
so called Smale spaces. On this setting he shows a relation of KMS states of $C^{*}$-algebras with Hölder equilibrium probabilities of Thermodynamic Formalism. Part of the formulation of this relation requires the use of a non trivial result by N. Haydn (see [10]). Later, the paper [11] by N. Haydn and D. Ruelle presents a shorter proof of the equivalence.

Here we consider similar problems but now on the symbolic space and the dynamics will be given by the shift. We will present a simplified proof of the equivalence mentioned above. The main result of this chapter is Theorem 18 on section 5. One can get a characterization of the equilibrium probability for a potential defined on the lattice $\{1,2, \ldots, d\}^{\mathbb{Z}-\{0\}}$ without using the Ruelle operator (which acts on the lattice $\{1,2, \ldots, d\}^{\mathbb{N}}$ ). The probability we get is invariant for the action of the shift $\tau$ acting on $\{1,2, \ldots, d\}^{\mathbb{Z}-\{0\}}$.

The proof of this result will take several subsequent sections.

In section 8 we show the relation of these probabilities with the KMS dynamical $C^{*}$-state on the $C^{*}$-Algebra associated to the groupoid defined by the homoclinic equivalence relation. On the initial sections we introduce several results which are necessary for the simplification of the final argument on section 8 .

We present several examples helping the reader on the understanding of the main concepts.

On [21] and also on the beginning of the book [1] it is explained the relation of equilibrium states of Thermodynamic Formalism with the corresponding concept in Statistical Physics. The role of KMS $C^{*}$-dynamical states on Quantum Statistical Physics is described on [4]. KMS $C^{*}$-dynamical states correspond to the DLR probabilities (see [6] for definition) in Statistical Mechanics.

In section 8 we present definitions and properties regarding the $C^{*}$-algebra we will consider here.

Working on the symbolic space helps to avoid several technicalities which are required in the case of the study of hyperbolic diffeomorphisms on manifolds (where one have to use stable foliation, the local product structure, etc...).

Our proof consider mainly potentials $A:\{1,2, \ldots, d\}^{\mathbb{Z}-\{0\}} \rightarrow \mathbb{R}$ which depend on a finite number of coordinates. The case of a general Hölder potential (more technical) can be obtained by adapting our reasoning but we will not address this question here.

On the papers [5] and [13] the authors consider among other things a relation of KMS probabilities with eigenprobabilities for the dual of the Ruelle operator (which are not necessarily invariant for the shift). This problem is analyzed on the lattice $\{1,2, . ., d\}^{\mathbb{N}}$ which is a different setting that the one we consider here. The equivalence relations are also not related. Despite 
some similarities that can be perceived in the statements of the main results obtained in the two settings we point out that the reasoning on the respective proofs are quite different.

Lecture 9 in [7] presents a brief introduction to $C^{*}$-Algebras and the KMS condition.

In [8] and [9] a relation of KMS states in a certain $C^{*}$-Algebra and eigenprobabilities of the dual of the Ruelle operator is considered.

In a different setting the paper 2] also considers the homoclinic equivalence relation.

\section{Conjugating homeomorphisms}

In this section $\Omega=\{1,2, \ldots, d\}^{\mathbb{Z}-\{0\}}$ and a general point $x$ on $\Omega$ is denoted as

$$
x=\left(\ldots, x_{-n}, \ldots, x_{-2}, x_{-1} \mid x_{1}, x_{2}, \ldots, x_{n}, \ldots\right),
$$

$x_{j} \in\{1,2, . ., d\}, j \in \mathbb{Z}$.

We consider the dynamics of the shift $\tau: \Omega \rightarrow \Omega$, that is,

$\tau\left(\ldots, x_{-n}, \ldots, x_{-2}, x_{-1} \mid x_{1}, x_{2}, \ldots, x_{n}, \ldots\right)=\left(\ldots, x_{-n}, \ldots, x_{-2}, x_{-1}, x_{1} \mid x_{2}, \ldots, x_{n}, \ldots\right)$

We also consider the usual metric $d$ on $\Omega$ which is defined in such way that for $x, y \in \Omega$ we set

$$
d(x, y)=2^{-N}
$$

$N \geq 0$, where for

$$
x=\left(\ldots, x_{-n}, \ldots, x_{-1} \mid x_{1}, . ., x_{n}, . .\right), y=\left(\ldots, y_{-n}, \ldots, y_{-1} \mid y_{1}, . ., y_{n}, . .\right),
$$

we have $x_{j}=y_{j}$, for all $j$, such that, $-N \leq j \leq N$ and, moreover $x_{N+1} \neq$ $y_{N+1}$, or $x_{-N-1} \neq y_{-N-1}$. Given $x, y$ as above we denote $\vartheta(x, y)=N$, therefore $\vartheta(x, y)=-\log _{2}(d(x, y))$.

Given $x, y \in \Omega$, we say that $x \sim y$ if

$$
\begin{aligned}
& \lim _{k \rightarrow+\infty} d\left(\tau^{k} x, \tau^{k} y\right)=0 \\
& \text { and } \\
& \lim _{k \rightarrow-\infty} d\left(\tau^{k} x, \tau^{k} y\right)=0 .
\end{aligned}
$$

This means there exists an $N \geq 0$ such that $x_{j}=y_{j}$ for $j>N$ and $j<-N$ (note that given $\epsilon>0$, there exists $n$ such that $2^{-n}<\epsilon \leq 2^{-n+1}$, and if $d(x, y)<\epsilon$, then $x$ and $y$ should coincide for coordinates smaller than $n)$. In 
other words, there are only a finite number of $i$ 's such that $x_{i} \neq y_{i}$. In this case we say that $x$ and $y$ are homoclinic.

In this way for large $k>0$ the strings for $\tau^{k}(x)=z^{x}$ and $\tau^{k}(y)=z^{y}$ are such that $z_{j}^{x}=z_{j}^{y}$ for $j$ in a large interval $j \in\{-R,-R+1, \ldots .,-1,1, \ldots . R-$ $1, R\}$, where $R$ is larger with $k$. Then, $\lim _{k \rightarrow+\infty} d\left(\tau^{k} x, \tau^{k} y\right)=0$.

$\sim$ is an equivalence relation and defines the groupoid $G \subset \Omega \times \Omega$ of pairs $(x, y)$ of elements which are related (see for instance [18], [19], [5] or [13]).

Let $\kappa(x, y)$ be the minimum $M$ as above. Therefore $x_{\kappa(x, y)} \neq y_{\kappa(x, y)}$ or $x_{-\kappa(x, y)} \neq y_{-\kappa(x, y)}$. Note that $\vartheta(x, y) \leq \kappa(x, y)$ and could be strictly less. Note that $\kappa(x, y)$ is defined just when $x \sim y$.

Example 1. For example in $\Omega=\{1,2\}^{\mathbb{Z}-\{0\}}$ take

$$
x=\left(\ldots, x_{-n}, \ldots, x_{-7}, 1,2,2,1,2,2 \mid 1,2,1,2,1,1, x_{7}, \ldots x_{n}, . .\right)
$$

and

$$
y=\left(\ldots, y_{-n}, \ldots, y_{-7}, 1,2,2,1,2,2 \mid 1,2,1,1,1,2, y_{7}, \ldots y_{n}, . .\right)
$$

where $x_{j}=y_{j}$ for $|j|>6=\kappa(x, y)$. In this case $d(x, y)=2^{-3}$ and $N=$ $\vartheta(x, y)=3$.

Given a Hölder function $U: \Omega \rightarrow \mathbb{R}$ it is easy to see that if $x$ and $y$ are homoclinic, then the following function is well defined

$$
V(x, y)=\sum_{n=-\infty}^{\infty}\left(U\left(\tau^{n}(x)\right)-U\left(\tau^{n}(y)\right)\right) .
$$

Indded, note that if $x \sim y$, they coincide for large $n$, then, there exists a constant $c$, such that, $d\left(\tau^{n}(x), \tau^{n}(y)\right) \leq c 2^{-n}$. If $U$ has Holder exponent $\alpha$, then, the sum converges absolutely because $\sum_{n}\left(2^{\alpha}\right)^{-n}<\infty$.

This function satisfies the property

$$
V(x, y)+V(y, z)=V(x, z)
$$

when $x \sim y \sim z$.

A function $V$ with this property will play an important role in some parts of our reasoning. We will not assume on the first part of this work that $V$ was obtained from a $U$ as above.

Now we will describe a certain class of conjugating homeomorphism for the relation $\sim($ see (1) $)$ described above.

Given two fixed points $x$ and $y$ ( $y$ in the class of $x$ ) we define the open set $\mathcal{O}_{(x, y)}=B_{\frac{1}{2^{\kappa(x, y)}}}(x)=\left\{z \in \Omega: d(x, z)<2^{-\kappa(x, y)+1}\right\}$. 
We will define for each such pair $(x, y)$ a conjugating homeomorphisms $\varphi_{(x, y)}$ which has domain on $\mathcal{O}_{(x, y)}$.

We denote for $m, n \in \mathbb{N}$

$$
\begin{gathered}
\overline{x_{-m} x_{-m+1} \ldots x_{-1} \mid x_{1} \ldots x_{n-1} x_{n}}= \\
\left\{z \in \Omega \mid z_{j}=x_{j}, j=-m,-m+1, \ldots,-1,1,2, \ldots, n-1, n\right\},
\end{gathered}
$$

and call it the cylinder determined by the finite string

$$
x_{-m} x_{-m+1} \ldots x_{-1} \mid x_{1} \ldots x_{n-1} x_{n} .
$$

We will say that a cylinder, or a string, is symmetric if $n=m$.

Note that given $x \sim y$

$$
\mathcal{O}_{(x, y)}=\overline{x_{-\kappa(x, y)} x_{-\kappa(x, y)+1} \ldots x_{-1} \mid x_{1} \ldots x_{\kappa(x, y)-1} x_{\kappa(x, y)}},
$$

and $\mathcal{O}_{(x, y)}$ is a symmetric cylinder.

Now we shall define the main kind of conjugating homeomorphisms that we will be using. Given $(x, y) \in G$, let $n=\kappa(x, y)$, we define a conjugating $\varphi=\varphi_{(x, y)}$ with domain

$$
\mathcal{O}_{(x, y)}=B_{\frac{1}{2^{n}}}(x)=\left\{z \in \Omega: d(x, z)<2^{-n+1}\right\}=\overline{x_{-n} x_{-n+1} \ldots x_{-1} \mid x_{1} \ldots x_{n-1} x_{n}},
$$

where $\varphi_{(x, y)}: \mathcal{O}_{(x, y)} \rightarrow B_{\frac{1}{2^{n}}}(y)$ is defined by the expression: $z$ of the form

$$
z=\left(\ldots z_{-n-2} z_{-n-1} \mathbf{x}_{-\mathbf{n}} \mathbf{x}_{-\mathbf{n}+\mathbf{1}} \ldots \mathbf{x}_{-\mathbf{1}} \mid \mathbf{x}_{\mathbf{1}} \ldots \mathbf{x}_{\mathbf{n}} z_{n+1} z_{n+2} \ldots\right)
$$

goes to

$$
\varphi_{(x, y)}(z)=\ldots z_{-n-2} z_{-n-1} \mathbf{y}_{-\mathbf{n}} \mathbf{y}_{-\mathbf{n}+\mathbf{1}} \ldots \mathbf{y}_{-\mathbf{1}} \mid \mathbf{y}_{\mathbf{1}} \ldots \mathbf{y}_{\mathbf{n}} z_{n+1} z_{n+2} \ldots
$$

We shall call these transformations the family of symmetric conjugating homeomorphisms. We shall denote by $S$ the set of symmetric conjugating homeomorphisms obtained by considering all pairs of related points $x$ and $y$.

Note that the homeomorphism $\varphi_{(x, y)}$ transforms the cylinder $O_{(x, y)}=$ $\overline{x_{-n} x_{-n+1} \ldots x_{-1} \mid x_{1} \ldots x_{n-1} x_{n}}$ in the cylinder $\overline{y_{-n} y_{-n+1} \ldots y_{-1} \mid y_{1} \ldots y_{n-1} y_{n}}$.

The graph of $\varphi_{(x, y)}$ is on $G$.

A more explicit formulation of the concept of symmetric conjugating homeomorphism will be presented on next section via expressions (6) and (7). 
Example 2. Consider

$$
x=(\ldots 112112222111 \mid 212122122211 \ldots)
$$

and

$$
y=(\ldots 112112222112 \mid 122122122211 \ldots)
$$

in this case $\kappa(x, y)=2$, and for $z$ of the form

$$
z=\left(\begin{array}{lllll}
\ldots z_{-4} z_{-3} & 1 & 1 \mid 2 & 1 & z_{3} z_{4} z_{5} \ldots
\end{array}\right)
$$

we get

$$
\varphi_{(x, y)}(z)=\left(\begin{array}{llll}
\ldots z_{-4} z_{-3} & 12 \mid 12 & z_{3} z_{4} z_{5} \ldots
\end{array}\right)
$$

It is easy to see that the family of symmetric conjugating homeomorphisms we define above has the following properties: given $x \sim y$

a) $\varphi_{(x, y)}: \mathcal{O}_{(x, y)} \subset \Omega \rightarrow \Omega$ is an homeomorphism over its image

b) $\varphi_{(x, y)}(x)=y$, and 0 .

c) $\lim _{k \rightarrow \infty} d\left(\tau^{k}(z), \tau^{k}\left(\varphi_{(x, y)}(z)\right)=0\right.$ and $\lim _{k \rightarrow-\infty} d\left(\tau^{k}(z), \tau^{k}\left(\varphi_{(x, y)}(z)\right)=\right.$

Item c) implies that $z$ and $\varphi_{(x, y)}(z)$ are on the same homoclinic class.

\section{$3 \quad C^{*}$-Gibbs states and Radon-Nikodym deriva- tive}

We consider the groupoid $G \subset \Omega \times \Omega$ of all pair of points which are related by the homoclinic equivalence relation.

We consider on $G$ the topology generated by sets of the form

$$
\left\{\left(z, \varphi_{(x, y)}(z)\right) \mid \text { where } z \in \mathcal{O}_{(x, y)} \text { with } x \sim y\right\} .
$$

This topology is Hausdorff (see [20]).

Now consider a continuous function $V: G \rightarrow \mathbb{R}$ such that

$$
V(x, y)+V(y, z)=V(x, z),
$$

for all related $x, y, z$. Note that this implies that $V(x, x)=0$ and $V(x, y)=$ $-V(y, x)$.

Here we call $V$ a modular function.

Under some other notation the function $\delta(x, y)=e^{V(x, y)}$ is called a modular function (or, a cocycle). 
Definition 3. Given a function $V: G \rightarrow \mathbb{R}$ as above we say that a probability measure $\alpha$ on $\Omega$ is a $C^{*}$-Gibbs probability with respect to the parameter $\beta \in \mathbb{R}$ and $V$, if for any $x \sim y$

$$
\int_{O_{(x, y)}} \exp \left(-\beta V\left(z, \varphi_{(x, y)}(z)\right)\right) f\left(\varphi_{(x, y)}(z)\right) d \alpha(z)=\int_{\varphi_{(x, y)}\left(O_{(x, y)}\right)} f(z) d \alpha(z),
$$

for every continuous function $f: \Omega \rightarrow \mathbb{C}$ (and conjugated homeomorphism $\left.\left(O_{(x, y)}, \varphi_{(x, y)}\right)\right)$.

We will show on section 8 a natural relation of this probability $\alpha$ with the $C^{*}$-dynamical state on a certain $C^{*}$-algebra. This is the reason for such terminology.

The above definition was taken from [20]. This is a version of the RenaultRadon-Nikodym condition (Def. 1.3.15 in [18]).

It is easy to see that the above definition is equivalent to say that: given any pair of finite strings

$$
x_{-n} x_{-n+1} \ldots x_{-1}, x_{1} \ldots x_{n-1} x_{n} \text { and } y_{-n} y_{-n+1} \ldots y_{-1} y_{1} \ldots y_{n-1} y_{n},
$$

$n \in \mathbb{N}$, the transformation

$$
\varphi: \overline{x_{-n} x_{-n+1} \ldots x_{-1} \mid x_{1} \ldots x_{n-1} x_{n}} \rightarrow \overline{y_{-n} y_{-n+1} \ldots y_{-1} \mid y_{1} \ldots y_{n-1} y_{n}}
$$

defined by the expression:

$$
\varphi(z)=\left(\ldots z_{-n-2} z_{-n-1} y_{-n} y_{-n+1} \ldots y_{-1} \mid y_{1} \ldots y_{n} z_{n+1} z_{n+2} \ldots\right),
$$

where

$$
z=\left(\ldots z_{-n-2} z_{-n-1} z_{-n} z_{-n+1} \ldots z_{-1} \mid z_{1} \ldots z_{n} z_{n+1} z_{n+2} \ldots\right),
$$

is such that for any continuous function $f: \overline{y_{-n} y_{-n+1} \ldots y_{n-1} y_{n}} \rightarrow \mathbb{R}$

$$
\int_{\frac{x_{-n} x_{-n+1} \ldots \mid \ldots x_{n-1} x_{n}}{}} e^{-\beta V(z, \varphi(z))} f(\varphi(z)) d \alpha(z)=\int_{\frac{y_{-n} y_{-n+1} \ldots \mid \ldots y_{n-1} y_{n}}{}} f(z) d \alpha(z) .
$$

Note in particulary that by taking $f=1$ we get

$$
\int \frac{}{x_{-n} x_{-n+1} \ldots \mid \ldots x_{n-1} x_{n}} e^{-\beta V(z, \varphi(z))} d \alpha(z)=\int \frac{}{y_{-n} y_{-n+1 \ldots \mid \ldots y_{n-1} y_{n}}} d \alpha(z) .
$$

In the moment we only consider symmetric conjugating homeomorphisms of the form (7). 
We will show on section 5 a relation of the $C^{*}$-Gibbs probabilities $\alpha$ with the Gibbs (equilibrium) probabilities of Thermodynamic Formalism.

In a more explicit formulation $\alpha$ is such that given any conjugating homeomorphism $\left(O_{(x, y)}, \varphi_{(x, y)}\right)$ of the form ([6) , and continuous function $f: \Omega \rightarrow \mathbb{C}$

$$
\begin{gathered}
\int_{O_{(x, y)}} e^{-\beta V\left(z, \varphi_{(x, y)}(z)\right)} f\left(\varphi_{(x, y)}(z)\right) d \alpha(z)= \\
\int_{O_{(x, y)}} e^{-\beta V\left(\left(\ldots z_{-n}, \ldots, z_{-1} \mid z_{1}, \ldots, z_{n}, \ldots\right),\left(\ldots z_{-n-1} y_{-n}, \ldots, y_{-1} \mid y_{1}, \ldots, y_{n}, z_{n+1}, \ldots\right)\right)} f\left(\varphi_{(x, y)}(z)\right) d \alpha(z)= \\
\int_{\varphi_{(x, y)}\left(O_{(x, y)}\right)} f(z) d \alpha(z)
\end{gathered}
$$

In this case, clearly the Radon-Nikodym derivative of the change of coordinates $\varphi$ is

$$
e^{-\beta V\left(\left(\ldots z_{-n}, \ldots, z_{-1} \mid z_{1}, \ldots, z_{n}, \ldots\right),\left(\ldots z_{-n-1} y_{-n}, \ldots, y_{-1} \mid y_{1}, \ldots, y_{n}, z_{n+1}, \ldots\right)\right)} .
$$

In order to simplify the notation sometimes on the text we will consider the value $\beta=1$.

We will consider a larger class of conjugating homeomorphisms.

Definition 4. Given $n$ and $m$ and pair of finite strings

$$
x_{-n} x_{-n+1} \ldots x_{-1}, x_{1} \ldots x_{m-1} x_{m} \text { and } y_{-n} y_{-n+1} \ldots y_{-1} y_{1} \ldots y_{n-1} y_{m},
$$

$n, m \in \mathbb{N}$, the transformation

$$
\varphi: \overline{x_{-n} x_{-n+1} \ldots x_{m-1} x_{m}} \rightarrow \overline{y_{-n} y_{-n+1} \ldots y_{m-1} y_{m}}
$$

defined by the expression:

$$
\varphi(z)=\left(\ldots z_{-n-2} z_{-n-1} \mathbf{y}_{-\mathbf{n}} \mathbf{y}_{-\mathbf{n}+\mathbf{1}} \ldots \mathbf{y}_{-\mathbf{1}} \mid \mathbf{y}_{\mathbf{1}} \ldots \mathbf{y}_{\mathbf{m}} z_{m+1} z_{m+2} \ldots\right),
$$

where

$$
z=\left(\ldots z_{-n-2} z_{-n-1} \mathbf{x}_{-\mathbf{n}} \mathbf{x}_{-\mathbf{n}+\mathbf{1}} \ldots \mathbf{x}_{-\mathbf{1}} \mid \mathbf{x}_{\mathbf{1}} \ldots \mathbf{x}_{\mathbf{m}} z_{m+1} z_{m+2} \ldots\right)
$$

is called a non-symmetric conjugating homeomorphism associated to the pair (11). 
Proposition 6 claims that if $\alpha$ is a $C^{*}$-Gibbs probability, then the relation (10) is satisfied for a bigger class of $\varphi$ transformations, i.e. not necessarily symmetric. Before that we shall provide the reader with an example of idea of the proof.

Example 5. Consider the non-symmetric conjugating homeomorphism $\varphi$ : $\overline{0 \mid 11} \rightarrow \overline{1 \mid 10}$ given by

$$
\varphi\left(\ldots z_{-3} z_{-2} 0 \mid 11 z_{3} \ldots\right)=\ldots z_{-3} z_{-2} 1 \mid 10 z_{3} \ldots
$$

we shall prove that if $\alpha$ is a $C^{*}$-Gibbs measure then relation (5) is valid for $\varphi$. This is actually straightforward, first divide the domain and image of the function into symmetric cylinders, and in these cylinders apply relation (10). So in this case consider $\varphi_{0}: \overline{00 \mid 11} \rightarrow \overline{01 \mid 10}$, and $\varphi_{1}: \overline{10 \mid 11} \rightarrow \overline{11 \mid 10}$ such that

$$
\varphi_{a}\left(\ldots z_{-3} a 0 \mid 11 z_{3} \ldots\right)=\left(\ldots z_{-3} a 1 \mid 10 z_{3} \ldots\right)
$$

for $a=0$ or $a=1$. Now notice that

$$
\begin{gathered}
\int \overline{\overline{0 \mid 11}} e^{-\beta V(x, \varphi(x))} f(\varphi(x)) d \alpha(x)= \\
\int_{\overline{00 \mid 11}} e^{-\beta V(x, \varphi(x))} f(\varphi(x)) d \alpha(x)+\int_{\overline{10 \mid 11}} e^{-\beta V(x, \varphi(x))} f(\varphi(x)) d \alpha(x)= \\
\int_{\overline{00 \mid 11}} e^{-\beta V\left(x, \varphi_{0}(x)\right)} f(\varphi(x)) d \alpha(x)+\int_{\overline{10 \mid 11}} e^{-\beta V\left(x, \varphi_{1}(x)\right)} f(\varphi(x)) d \alpha(x) \stackrel{(10)}{=} \\
\int_{\frac{01 \mid 10}{=}} f(x) d \alpha(x)+\int_{\overline{11 \mid 10}} f(x) d \alpha(x)=\int_{\overline{1 \mid 10}} f(x) d \alpha(x) .
\end{gathered}
$$

This claim proves that relation (10) is valid for this conjugating.

Proposition 6. Assume $\alpha$ is $C^{*}$-Gibbs for $V$ as in (10), then for any non-simmetric homeomorphism $(\varphi, \mathcal{O})$, as defined on (13), we have that for $n, m \in \mathbb{N}$, the transformation

$$
\begin{gathered}
\int_{\frac{x_{-n} x_{-n+1} \ldots x_{-1} \mid x_{1} \ldots x_{m-1} x_{m}}{}} e^{-\beta V(z, \varphi(z))} f(\varphi(z)) d \alpha(z)= \\
\int_{\mathcal{O}} e^{-\beta V\left(\left(\ldots z_{-n-1} z_{-n}, \ldots, z_{-1} \mid z_{1}, \ldots, z_{m}, z_{m+1} \ldots\right),\left(\ldots z_{-n-1} y_{-n}, \ldots, y_{-1} \mid y_{1}, \ldots, y_{m}, z_{m+1}, \ldots\right)\right)} f(\varphi(z)) d \alpha(z)= \\
\int_{\frac{y_{-n} y_{-n+1} \ldots y_{-1} \mid y_{1} \ldots y_{m-1} y_{m}}{}} f(z) d \alpha(z) .
\end{gathered}
$$


We leave the proof (which is similar to the reasoning of example 5) for the reader.

As a particular case we get

$$
\int_{\frac{\mid x_{1} \ldots x_{m}}{}} e^{-\beta V(z, \varphi(z))} f(\varphi(z)) d \alpha(z)=\int_{\frac{y_{1} \ldots y_{m}}{}} f(z) d \alpha(z) .
$$

for given $\overline{\mid x_{1} \ldots x_{m}}, \overline{\mid y_{1} \ldots y_{m}}$ and the corresponding conjugating homeomorphism $\varphi$.

It is possible to consider more general forms of conjugating homeomorphisms as described on the next example.

Example 7. Consider the homeomorphism $\varphi: \overline{112 \mid 2} \rightarrow \overline{1 \mid 122}$ given by

$$
\varphi\left(\ldots z_{-4} 112 \mid 2 z_{2} z_{3} z_{4} \ldots\right)=\left(\ldots z_{-4} z_{2} z_{3} 1 \mid 122 z_{4} \ldots\right) .
$$

Note that $\overline{112 \mid 2}$ is translation by $\tau^{-2}$ of the set $\overline{1 \mid 122}$.

$A s$ in the previous example we will prove that if $\alpha$ is a $C^{*}$-Gibbs probability then relation (10) is also valid for such $\varphi$ and $\mathcal{O}=\overline{112 \mid 2}$. First consider the conjugating homeomorphisms, $\varphi_{1}, \varphi_{2}, \varphi_{3}$ and $\varphi_{4}$, given by

$$
\begin{aligned}
& \varphi_{1}\left(\ldots z_{-4} 112 \mid 211 z_{4} \ldots\right)=\left(\ldots z_{-4} 111 \mid 122 z_{4} \ldots\right), \\
& \varphi_{2}\left(\ldots z_{-4} 112 \mid 212 z_{4} \ldots\right)=\left(\ldots z_{-4} 121 \mid 122 z_{4} \ldots\right), \\
& \varphi_{3}\left(\ldots z_{-4} 112 \mid 221 z_{4} \ldots\right)=\left(\ldots z_{-4} 211 \mid 122 z_{4} \ldots\right), \\
& \varphi_{4}\left(\ldots z_{-4} 112 \mid 2 \mathbf{2 2} z_{4} \ldots\right)=\left(\ldots z_{-4} 221 \mid 122 z_{4} \ldots\right) .
\end{aligned}
$$

Therefore we have that

$$
\begin{gathered}
\int_{\overline{112 \mid 2}} e^{V(x, \varphi(x))} f(\varphi(x)) d \alpha(x)= \\
\int_{\overline{112 \mid 211}} e^{V(x, \varphi(x))} f(\varphi(x))+\int_{\overline{112 \mid 212}} e^{V(x, \varphi(x))} f(\varphi(x))+ \\
\int_{\overline{112 \mid 221}} e^{V(x, \varphi(x))} f(\varphi(x))+\int_{\overline{112 \mid 222}} e^{V(x, \varphi(x))} f(\varphi(x))= \\
\int_{\overline{112 \mid 211}} e^{V\left(x, \varphi_{1}(x)\right)} f\left(\varphi_{1}(x)\right)+\int_{\overline{112 \mid 212}} e^{V\left(x, \varphi_{2}(x)\right)} f\left(\varphi_{2}(x)\right)+ \\
\int_{\frac{112 \mid 221}{}} e^{V\left(x, \varphi_{3}(x)\right)} f\left(\varphi_{3}(x)\right)+\int_{\overline{112 \mid 222}} e^{V\left(x, \varphi_{4}(x)\right)} f\left(\varphi_{4}(x)\right)=
\end{gathered}
$$




$$
\begin{gathered}
\int_{\overline{11|| 122}} f(x)+\int_{\overline{121 \mid 122}} f(x)+\int_{\overline{211 \mid 122}} f(x)+\int_{\overline{221 \mid 122}} f(x)= \\
\int_{\overline{1 \mid 122}} f d \alpha(x)
\end{gathered}
$$

where some of the $d \alpha$ where omitted. Since we proved that

$$
\int_{\overline{112 \mid 2}} e^{V(x, \varphi(x))} f(\varphi(x)) d \alpha(x)=\int_{\overline{1 \mid 122}} f d \alpha(x)
$$

for any continuous function $f$ then we have that relation (10) is satisfied.

In analogous way as in last example one can define a conjugating $\varphi$ such that

$\varphi: \overline{x_{-n} \ldots \mathbf{x}_{-\mathbf{r}} \ldots \mathbf{x}_{-\mathbf{1}} \mid x_{1} \ldots x_{m}} \rightarrow \overline{x_{-n} x_{-n+1} \ldots x_{-r-1} \mid \mathbf{x}_{-\mathbf{r}} \ldots \mathbf{x}_{-\mathbf{1}} x_{1} \ldots x_{m}}$.

We will consider such transformation $\varphi$ in the next result.

Proposition 8. Assume $\alpha$ is $C^{*}$-Gibbs for $V$ as in (10), then for $n, m \in \mathbb{N}$, and $0<r$, such that, $r \leq n$, we get

$$
\begin{aligned}
& \int_{\overline{x_{-n} x_{-n+1} \ldots x_{-r-1} \mathbf{x}_{-\mathbf{r}} \mathbf{x}_{-\mathbf{r}+1} \ldots \mathbf{x}_{-1} \mid x_{1} \ldots x_{m-1} x_{m}}} e^{-\beta V(z, \varphi(z))} f(\varphi(z)) d \alpha(z)= \\
& \int \frac{}{x_{-n} x_{-n+1} \ldots x_{-r-1} \mid \mathbf{x}_{-\mathbf{r}} \mathbf{x}_{-\mathbf{r}+1} \ldots \mathbf{x}_{-1} \mathbf{x}_{\mathbf{1}} \ldots x_{m-1} x_{m}} f(z) d \alpha(z),
\end{aligned}
$$

where $\varphi$ is of the form (13).

Proof: The proof is similar to the reasoning of example 7. One just has to consider the homeomorphisms

$$
\begin{aligned}
& \varphi\left(\ldots z_{-n-r-1} z_{-n-r} \ldots z_{-n-1} x_{-n} x_{-n+1} \ldots x_{-1} \mid x_{1} \ldots x_{m-1} x_{m} \mathbf{z}_{\mathbf{m}+\mathbf{1}} \ldots \mathbf{z}_{\mathbf{m}+\mathbf{r}} z_{m+r+1} \ldots\right)= \\
& \left(\ldots z_{-n-r} \mathbf{z}_{\mathbf{m}+\mathbf{1}} \ldots \mathbf{z}_{\mathbf{n}+\mathbf{r}} x_{-n} x_{-n+1} \ldots x_{-r-1} \mid x_{-r} x_{-r+1} \ldots x_{-1} x_{1} \ldots x_{m-1} x_{m} z_{n+r+1} \ldots\right) .
\end{aligned}
$$

Note that

$$
\frac{\tau^{-r}\left(\overline{x_{-n} x_{-n+1} \ldots x_{-1} \mid x_{1} \ldots x_{m-1} x_{m}}\right)=}{x_{-n} x_{-n+1} \ldots x_{-r-1} \mid x_{-r} x_{-r+1} \ldots x_{-1} x_{1} \ldots x_{m-1} x_{m}} .
$$

We want to show that $\alpha$ is $C^{*}$-Gibbs for $V$, then, the pullback $\rho=\tau^{*}(\alpha)$ is also $C^{*}$-Gibbs for $V$.

The next example will help to understand the main reasoning for the proof of the above claim. 
Example 9. Suppose $V(x, y)$ is defined when $x \sim y$. Assume that for all $x, y$ on the groupoid we have that $V(x, y)=V(\tau(x), \tau(y))$.

Given $\alpha$ consider the pull back $\rho=\tau^{*}(\alpha)$.

Consider

$$
\varphi: \overline{11 \mid 21} \rightarrow \overline{21 \mid 12}
$$

where

$$
\varphi\left(\ldots x_{-4} x_{-3} 11 \mid 21 x_{3} x_{4} \ldots\right)=\left(\ldots x_{-4} x_{-3} 21 \mid 12 x_{3} x_{4} \ldots\right),
$$

and

$$
\varphi_{1}: \overline{112 \mid 1} \rightarrow \overline{211 \mid 2}
$$

where

$$
\varphi_{1}\left(\ldots x_{-5} x_{-4} 112 \mid 1 x_{2} x_{3} \ldots\right)=\left(\ldots x_{-5} x_{-4} 211 \mid 2 x_{2} x_{3} \ldots\right) .
$$

If for any continuous function $g$ we have that

$$
\int_{\overline{11 \mid 21}} e^{V(x, \varphi(x))} g(\varphi(x)) d \alpha(x)=\int_{\overline{21 \mid 12}} g(x) d \alpha(x),
$$

then, for any continuous function $f$ we have that

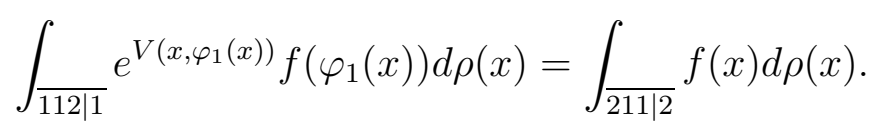

In fact both properties are equivalent.

Note first that $\varphi_{1} \circ \tau=\tau \circ \varphi$.

Moreover, $V\left(\tau(x), \varphi_{1}(\tau(x))=V\left(\tau(x), \tau\left(\varphi_{1}(x)\right)=V\left(x, \varphi_{1}(x)\right)\right.\right.$ by hypothesis.

Therefore,

$$
\begin{gathered}
\int \overline{112 \mid 1} e^{V\left(x, \varphi_{1}(x)\right)} f\left(\varphi_{1}(x)\right) d \rho(x)= \\
\int I_{\overline{112 \mid 1}}(x) e^{V\left(x, \varphi_{1}(x)\right)} f\left(\varphi_{1}(x)\right) d \rho(x)= \\
\int I_{\overline{112 \mid 1}}(\tau(x)) e^{V\left(\tau(x), \varphi_{1}(\tau(x))\right)} f\left(\varphi_{1}(\tau(x))\right) d \alpha(x)= \\
\int I_{\overline{112 \mid 1}}(\tau(x)) e^{V\left(x, \varphi_{1}(x)\right)} f\left(\varphi_{1}(\tau(x))\right) d \alpha(x)= \\
\left.\int I_{\overline{112 \mid 1}}(\tau(x)) e^{V\left(x, \varphi_{1}(x)\right)} f(\tau(\varphi(x)))\right) d \alpha(x)= \\
\left.\int I_{\overline{11 \mid 21}}(x) e^{V\left(x, \varphi_{1}(x)\right)} f(\tau(\varphi(x)))\right) d \alpha(x)=
\end{gathered}
$$




$$
\begin{gathered}
\left.\int \overline{11 \mid 21} e^{V\left(x, \varphi_{1}(x)\right)} f(\tau(\varphi(x)))\right) d \alpha(x)= \\
\int \frac{}{21 \mid 12} f(\tau(x)) d \alpha(x)= \\
\int I \overline{21 \mid 12}(x) f(\tau(x)) d \alpha(x)= \\
\int I \frac{}{21 \mid 12}\left(\tau^{-1} \circ \tau\right)(x) f(\tau(x)) d \alpha(x)= \\
\int I_{\overline{21 \mid 12}}\left(\tau^{-1}(x)\right) f(x) d \rho(x)= \\
\int_{\overline{211 \mid 2}} f(x) d \rho(x) .
\end{gathered}
$$

Above we took $g=f \circ \tau$.

From the above reasoning we get that both properties are equivalent.

Proposition 10. If $\alpha$ is $C^{*}$-Gibbs for $V$, and $V(x, y)=V(\tau(x), \tau(y))$, for all $x, y \in G$, then, the pull back $\rho=\tau^{*}(\alpha)$ is also $C^{*}$-Gibbs for $V$.

Proof: Suppose $\alpha$ is $C^{*}$-Gibbs for $V$.

The reasoning of the proof is just a generalization of the argument used on last example.

Consider for $r, s>0$

$$
\varphi: \overline{a_{-r} \ldots a_{-1} \mid a_{1} a_{2} \ldots a_{s}} \rightarrow \overline{b_{-r} \ldots b_{-1} \mid b_{1} b_{2} \ldots b_{s}},
$$

where

$$
\begin{gathered}
\varphi\left(\ldots x_{-r+2} x_{-r+1} a_{-r} \ldots a_{-1} \mid a_{1} a_{2} \ldots a_{s} x_{s+1} x_{s+2} \ldots\right)= \\
\left(\ldots x_{-r+2} x_{-r+1} b_{-r} \ldots b_{-1} \mid b_{1} b_{2} \ldots b_{s} x_{s+1} x_{s+2} \ldots\right),
\end{gathered}
$$

and

$$
\varphi_{1}: \overline{a_{-r} \ldots a_{-1} a_{1} \mid a_{2} \ldots a_{s}} \rightarrow \overline{b_{-r} \ldots b_{-1} b_{1} \mid b_{2} \ldots b_{s}}
$$

where

$$
\begin{gathered}
\varphi\left(\ldots x_{-r+2} x_{-r+1} a_{-r} \ldots a_{-1} a_{1} \mid a_{2} \ldots a_{s} x_{s+1} x_{s+2} \ldots\right)= \\
\left(\ldots x_{-r+2} x_{-r+1} b_{-r} \ldots b_{-1} b_{1} \mid b_{2} \ldots b_{s} x_{s+1} x_{s+2} \ldots\right),
\end{gathered}
$$

Adapting the argument of last example one can easily show that if for any continuous function $g$ we have that

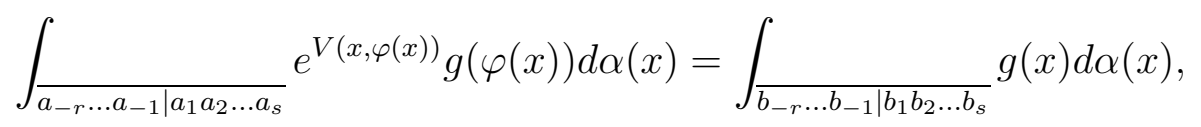


then, for any continuous function $f$ we have that

$$
\int_{\frac{a_{-r} \ldots a_{-1} a_{1} \mid a_{2} \ldots a_{s}}{}} e^{V\left(x, \varphi_{1}(x)\right)} f\left(\varphi_{1}(x)\right) d \rho(x)=\int_{\frac{b_{-r} \ldots b_{-1} b_{1} \mid b_{2} \ldots b_{s}}{}} f(x) d \rho(x) .
$$

As $\alpha$ is $C^{*}$-Gibbs for $V$, then (18) is true for any $f$. From (18) it follows that $\rho$ is $C^{*}$-Gibbs for $V$.

We point out that it is equivalent to ask the $C^{*}$-Gibbs property for $V$ taking symmetric cylinders or taking not symmetric cylinders (this is implicit on the proof of Proposition 8 ).

\section{Modular functions and potentials}

As we mentioned before given a Hölder function $U: \Omega \rightarrow \mathbb{R}$ there is a natural way (described by (2)) to get a continuous function $V$ satisfying the property (4).

We suppose now that $V$ is such that $V(x, y)=\sum_{k=-\infty}^{\infty}\left[U\left(\tau^{k}(x)-U\left(\tau^{k}(y)\right]\right.\right.$, when $x \sim y$, where $U: \Omega \rightarrow \mathbb{R}$ is Hölder (see (2) ). The function $U$ will sometimes be called a potential. We shall also suppose that $U$ is a finite range potential, or equivalently that it depends on a finite number of positive coordinates, that is, there is $k \in \mathbb{N}$ and a function $f:\{1, \ldots, d\}^{k} \rightarrow \mathbb{R}$, such that, for all $x \in \Omega$ we get

$$
U(x)=U\left(\ldots x_{-n} x_{-n+1} \ldots x_{-2} x_{-1} \mid x_{1} x_{2} \ldots x_{m-1} x_{m} \ldots\right)=f\left(x_{1}, x_{2}, \ldots, x_{k}\right),
$$

for this fixed $f$ and $k>0$, where $U: \Omega \rightarrow \mathbb{R}$. In this case we say that $U$ depends on $k$ coordinates.

Note that such $V$ satisfies $V(x, y)=V(\tau(x), \tau(y))$ and then Proposition 10 can be applied.

Remark 1: By abuse of language we can write $U:\{1,2, . ., d\}^{\mathbb{N}} \rightarrow \mathbb{R}$.

If $x \sim y$ it isn't hard to see that there is a finite $M>0$, such that,

$$
V(x, y)=\sum_{k=-\infty}^{\infty}\left[U\left(\tau^{k}(x)\right)-U\left(\tau^{k}(y)\right)\right]=\sum_{k=-M}^{M}\left[U\left(\tau^{k}(x)\right)-U\left(\tau^{k}(y)\right)\right] .
$$

In this way, if $z \sim \varphi(z)$, then,

$$
V(z, \varphi(z))=\sum_{k=-M}^{M} U\left(\tau^{k}(z)\right)-\sum_{k=-M}^{M} U\left(\tau^{k}(\varphi(z))=\sum_{k=-M}^{M}\left[U \left(\tau^{k}(z)-U\left(\tau^{k}(\varphi(z))\right]\right.\right.\right.
$$


Therefore, in this case, equation (10) means

$$
\begin{gathered}
\int_{x_{-n}, \ldots, x_{-1} \mid x_{1}, x_{2}, \ldots, y_{n}} e^{\sum_{k=-M}^{M} U\left(\tau^{k}(\varphi(z))\right]-\sum_{k=-M}^{M} U\left(\tau^{k}(z)\right)} f(\varphi(z)) d \alpha(z)= \\
\int_{\frac{y_{-n}, \ldots, y_{-1} \mid y_{1}, y_{2}, \ldots, y_{n}}{}} f(z) d \alpha(z)
\end{gathered}
$$

If $\alpha$ is $C^{*}$-Gibbs for $V$, and $V(z, \varphi(z))=\sum_{k=-\infty}^{+\infty} U(z)-U(\varphi(z))$ we also say by abuse of language that $\alpha$ is $C^{*}$-Gibbs for $U: \Omega \rightarrow \mathbb{R}$.

Definition 11. Given a function $V: G \rightarrow \mathbb{R}, V(x, y)=\sum_{k=-\infty}^{\infty}\left[U\left(\tau^{k}(x)\right)-\right.$ $\left.U\left(\tau^{k}(y)\right)\right]$, with $U$ of Hölder class, we say that a probability measure $\alpha$ on $\Omega$ is the quasi $C^{*}$-Gibbs probability with respect to the parameter $\beta \in \mathbb{R}$ and $U$, if there exists constants $d_{1}>0$ and $d_{2}>0$, such that, for any $x \sim y$ and any $O_{(x, y)}$,

$$
\begin{gathered}
d_{1} \int_{O_{(x, y)}} \exp \left(-\beta V\left(z, \varphi_{(x, y)}(z)\right)\right) g\left(\varphi_{(x, y)}(z)\right) d \alpha(z) \leq \\
\int_{\varphi_{(x, y)}\left(O_{(x, y)}\right)} g(z) d \alpha(z) \leq d_{2} \int_{O_{(x, y)}} \exp \left(-\beta V\left(z, \varphi_{(x, y)}(z)\right)\right) g\left(\varphi_{(x, y)}(z)\right) d \alpha(z)
\end{gathered}
$$

for every every continuous function $g: \Omega \rightarrow \mathbb{C}$ (and symmetric conjugated homeomorphism $\left(O_{(x, y)}, \varphi_{(x, y)}\right)$.

In the same way as before one can extend the above property for symmetric conjugated homeomorphisms to non symmetric conjugated homeomorphisms.

A $C^{*}$-Gibbs probability is a quasi $C^{*}$-Gibbs probability.

We say that a potential $\tilde{U}:\{1,2 . ., d\}^{\mathbb{N}} \rightarrow \mathbb{R}$ - which depends on a finite number of coordinates - is normalized, if for $k$ large enough and for any $\left(x_{1}, x_{2}, . ., x_{k}\right)$ we get $\sum_{j=1}^{d} e^{\tilde{U}\left(j, x_{1} \ldots, x_{k-1}\right)}=1$ - in particularly, we get $e^{\tilde{U}(x)}=$ $e^{\tilde{U}\left(x_{1} \ldots, x_{k}\right)}<1$ for all $x=\left(x_{1}, x_{2}, \ldots\right) \in\{1,2, \ldots, d\}^{\mathbb{N}}$.

From this follows that for any $w=\left(w_{1}, w_{2}, \ldots, w_{m}, \ldots\right) \in\{1,2, \ldots, d\}^{\mathbb{N}}$ and $n \in \mathbb{N}$,

$$
\sum_{z_{1}, z_{2}, . ., z_{n}=1}^{d} e^{\sum_{j=0}^{n-1} \tilde{U}\left(\sigma^{j}\left(z_{1}, z_{2}, . ., z_{n}, w_{1}, w_{2}, w_{3}, \ldots, w_{m}, \ldots\right)\right.}=1
$$


where $\sigma$ is the shift acting on $\{1,2, . ., d\}^{\mathbb{N}}$.

Suppose for such $U$ that $\alpha$ is quasi $C^{*}$-Gibbs for $U$ (satisfies the double inequality (21) for any continuous $g$ ). This implies in particular that there exist $d_{1}, d_{2}>0$, such that, for any cylinders of the form $\overline{\mid x_{1}^{0}, x_{2}^{0}, \ldots x_{s}^{0}}$ and $\overline{\mid y_{1}^{0}, y_{2}^{0}, \ldots y_{s}^{0}}$, and a function $\varphi$, such that,

$$
\begin{gathered}
d_{1} \int_{\frac{\mid x_{1}^{0}, x_{2}^{0}, \ldots x_{s}^{0}}{}} e^{-\beta V(z, \varphi(z))} g\left(\varphi_{(x, y)}(z)\right) d \alpha(z) \leq \\
\int \frac{y_{1}^{0}, y_{2}^{0}, \ldots y_{s}^{0}}{g} g(z) d \alpha(z) \leq d_{2} \int_{\frac{\mid x_{1}^{0}, x_{2}^{0}, \ldots x_{s}^{0}}{}} e^{-\beta V(z, \varphi(z))} g\left(\varphi_{(x, y)}(z)\right) d \alpha(z),
\end{gathered}
$$

where $\varphi_{(x, y)}$ is the associated conjugating homeomorphism, such that,

$$
\varphi_{(x, y)}:\left(\overline{\mid x_{1}^{0}, x_{2}^{0}, \ldots x_{s}^{0}}\right) \rightarrow \overline{\mid y_{1}^{0}, y_{2}^{0}, \ldots y_{s}^{0}}
$$

Example 12. Consider the homeomorphism $\varphi: \overline{112 \mid 2} \rightarrow \overline{\mid 1122}$ given by

$$
\varphi\left(\ldots z_{-4} 112 \mid 2 z_{2} z_{3} z_{4} \ldots\right)=\left(\ldots z_{-4} z_{2} z_{3} z_{4} \mid 1122 z_{5} \ldots\right) .
$$

Note that $\overline{112 \mid 2}$ is translation by $\tau^{-3}$ of the set $\overline{\mid 1122}$.

Consider the conjugating homeomorphisms, $\varphi_{1}, \varphi_{2}, \varphi_{3}$ and $\varphi_{4}$, given by

$$
\begin{aligned}
& \varphi_{1}\left(\ldots z_{-4} 112 \mid 2 \mathbf{1 1} z_{4} \ldots\right)=\left(\ldots z_{-4} \mathbf{1 1} \mid 1122 z_{5} \ldots\right), \\
& \varphi_{2}\left(\ldots z_{-4} 112 \mid 2 \mathbf{1 2} z_{4} \ldots\right)=\left(\ldots z_{-4} \mathbf{1 2} \mid 1122 z_{5} \ldots\right), \\
& \varphi_{3}\left(\ldots z_{-4} 112 \mid 2 \mathbf{2 1} z_{4} \ldots\right)=\left(\ldots z_{-4} \mathbf{2 1} \mid 1122 z_{5} \ldots\right), \\
& \varphi_{4}\left(\ldots z_{-4} 112 \mid 2 \mathbf{2 2} z_{4} \ldots\right)=\left(\ldots z_{-4} 22 \mid 1122 z_{5} \ldots\right) .
\end{aligned}
$$

Suppose $\alpha$ is quasi-C* Gibbs and satisfies (21).

Therefore,

$$
\begin{gathered}
\int_{\overline{112 \mid 2}} e^{V(x, \varphi(x))} f(\varphi(x)) d \alpha(x)= \\
\int_{\overline{112 \mid 211}} e^{V(x, \varphi(x))} f(\varphi(x))+\int_{\overline{112 \mid 212}} e^{V(x, \varphi(x))} f(\varphi(x))+ \\
\int_{\overline{112 \mid 221}} e^{V(x, \varphi(x))} f(\varphi(x))+\int_{\overline{112 \mid 222}} e^{V(x, \varphi(x))} f(\varphi(x))= \\
\int_{\overline{112 \mid 211}} e^{V\left(x, \varphi_{1}(x)\right)} f\left(\varphi_{1}(x)\right)+\int_{\overline{112 \mid 212}} e^{V\left(x, \varphi_{2}(x)\right)} f\left(\varphi_{2}(x)\right)+
\end{gathered}
$$




$$
\begin{gathered}
\int \frac{1}{112 \mid 221} e^{V\left(x, \varphi_{3}(x)\right)} f\left(\varphi_{3}(x)\right)+\int_{\frac{112 \mid 222}{d_{1}}} e^{V\left(x, \varphi_{4}(x)\right)} f\left(\varphi_{4}(x)\right) \leq \\
\left.\frac{1}{d_{1}} \int_{\frac{1122}{\mid 1122}} f(x)+\int_{\frac{12 \mid 1122}{21 \mid 1122}} f(x)+\int_{\frac{22 \mid 1122}{2}} f(x)\right]=
\end{gathered}
$$

where some of the d $\alpha$ where omitted. We proved that

$$
\int_{\overline{112 \mid 2}} e^{V(x, \varphi(x))} f(\varphi(x)) d \alpha(x) \leq \frac{1}{d_{1}} \int_{\overline{\mid 1122}} f d \alpha(x),
$$

for any measurable function $f$.

Taking $f=1$, we get that

$$
\int_{\overline{112 \mid 2}} e^{V(x, \varphi(x))} d \alpha(x) \leq \frac{1}{d_{1}} \int_{\overline{\mid 1122}} d \alpha(x) .
$$

As $e^{V(x, \varphi(x))}$ is strictly positive we get that if $\alpha(\overline{\mid 1122})=0$, then, $\alpha(\overline{112 \mid 2})=$ 0 .

Using the inequality for $d_{2}$ in (21) we get in a similar way that if $\alpha(\overline{112 \mid 2})=$ 0 , then, $\alpha(\overline{\mid 1122})=0$.

One can also show that

$$
\int_{\overline{\mid 1122}} d \alpha(x) \leq d_{2} \int_{\overline{112 \mid 2}} e^{V(x, \varphi(x))} d \alpha(x)
$$

Proposition 13. Suppose $\alpha$ is quasi-C $C^{*}$-Gibbs for a potential $U$ that depends on finite coordinates, then

$$
\alpha\left(\overline{a_{-r} \ldots a_{-1} \mid a_{1} a_{2} \ldots a_{s}}\right)>0,
$$

if and only if,

$$
\alpha\left(\mid \overline{a_{-r} \ldots a_{-1} a_{1} a_{2} \ldots a_{s}}\right)>0 .
$$

Moreover, there exist $b_{1}>0, b_{2}>0$, such that, for any cylinder set of the form $\overline{a_{-r} \ldots a_{-1} \mid a_{1} a_{2} \ldots a_{s}}$ we get

$$
\begin{gathered}
b_{1} \alpha\left(\overline{a_{-r} \ldots a_{-1} \mid a_{1} a_{2} \ldots a_{s}}\right) \leq \alpha\left(\overline{\mid a_{-r} \ldots a_{-1} a_{1} a_{2} \ldots a_{s}}\right) \leq \\
b_{2} \alpha\left(\overline{a_{-r} \ldots a_{-1} \mid a_{1} a_{2} \ldots a_{s}}\right) .
\end{gathered}
$$


Proof: We left the proof for the reader which is an adaptation of the reasoning of Example 12.

The next result shows that we can always consider normalized potentials (see Theorem 2.2 in [16] for general results) on the definition of quasi $C^{*}$ Gibbs probability.

Theorem 14. Suppose the probability $\alpha$ on $\Omega$ is $C^{*}$-Gibbs for Hölder potential $U$. Assume, $X: \Omega \rightarrow \mathbb{R}$ is such that $X=U+g-g \circ \tau+\lambda$, where $g: \Omega \rightarrow \mathbb{R}$ is a Hölder continuous function and $\lambda$ a constant, then $\alpha$ is quasi $C^{*}$-Gibbs for $X$.

Proof: Suppose that for any continuous $f$ we have

$$
\begin{gathered}
\int_{O_{x, y}} e^{\beta \sum_{k=-\infty}^{\infty} U\left(\tau^{k}(\varphi(z))\right)-U\left(\tau^{k}(z)\right)} f(\varphi(z)) d \alpha(z)= \\
\int_{\varphi\left(O_{(x, y)}\right)} f(z) d \alpha(z)
\end{gathered}
$$

Note that

$$
\sum_{k=-\infty}^{\infty}\left[g\left(\tau^{k}(z)\right)-g\left(\tau^{k}(\varphi(z))\right)\right]
$$

is limited since $g$ is Hölder, actually the summation is absolutely convergent by the same reason. The same can be said of

$$
\sum_{k=-\infty}^{\infty}\left[g\left(\tau^{k+1}(z)\right)-g\left(\tau^{k+1}(\varphi(z))\right)\right]
$$

and of

$$
\sum_{k=-\infty}^{\infty}\left[U\left(\tau^{k}(z)\right)-U\left(\tau^{k} \varphi(z)\right)\right]
$$

The absolute convergence allow us to sum the quantities above in any order, the resulting sum is limited since each of the above quantities are.

Therefore,

$$
\begin{aligned}
& \sum_{k=-\infty}^{\infty}\left[X\left(\tau^{k}(\varphi(z))\right)-X\left(\tau^{k}(z)\right)\right]= \\
& {\left[\sum_{k=-\infty}^{\infty} U\left(\tau^{k}(\varphi(z))\right]-U\left(\tau^{k}(z)\right)\right]+}
\end{aligned}
$$




$$
\begin{gathered}
{\left[\sum_{k=-\infty}^{\infty} g\left(\tau^{k}(\varphi(z))-g\left(\tau^{k}(z)\right)\right]-\right.} \\
{\left[\sum_{k=-\infty}^{\infty} g\left(\tau^{k+1}(\varphi(z))\right)-g\left(\tau^{k+1}(z)\right)\right]}
\end{gathered}
$$

is bounded above and below by constants which do not depend on $x \sim y$, $O_{x, y}$ and corresponding $\varphi_{x, y}$.

Then, $\alpha$ is quasi $C^{*}$-Gibbs for $X$.

By Proposition 1.2 in [16] given a Hölder potential $U: \Omega \rightarrow \mathbb{R}$, one can find $W$ depending on positive coordinates $(1,2,3, . ., n, \ldots) \in\{1,2, \ldots, d\}^{\mathbb{N}}$ and a continuous function $v: \Omega \rightarrow \mathbb{R}$ (which depends on finite coordinates), such that, $W=U+v-v \circ \tau$.

The function $V$ is Hölder and then last theorem can be applied.

More precisely, there exist $\tilde{W}:\{1,2, \ldots, d\}^{\mathbb{N}} \rightarrow \mathbb{R}$ an $r$, such that,

$$
\begin{gathered}
W\left(\ldots x_{-n-1} x_{-n} x_{-n+1} \ldots x_{-1} \mid x_{1} \ldots x_{m} x_{m+1} \ldots\right)= \\
\tilde{W}\left(x_{1} \ldots x_{m} x_{m+1} \ldots\right)=K\left(x_{1} \ldots x_{r}\right),
\end{gathered}
$$

for a certain function $K:\{1,2, \ldots, d\}^{r} \rightarrow \mathbb{R}$.

The bottom line is: from Theorem 2.2 in [16], given such $\tilde{W}$ one can find, $u$ and positive constant $\lambda$, such that, $\tilde{W}=\tilde{U}+u-u \circ \tau+\lambda$. Moreover, $\tilde{U}:\{1,2, \ldots, d\}^{\mathbb{N}} \rightarrow \mathbb{R}$ and $u:\{1,2, \ldots, d\}^{\mathbb{N}} \rightarrow \mathbb{R}$ both depend on a finite number of coordinates.

Remark 2: Therefore, from Theorem 14 if $\alpha$ is $C^{*}$-Gibbs for a Hölder potential $U: \Omega \rightarrow \mathbb{R}$, which depends on a finite number of coordinates, we can assume that $\alpha$ is quasi- $C^{*}$-Gibbs for another potential, denoted $\tilde{U}$, which is normalized and depending on a finite number of coordinates.

By abuse of language one can write $\tilde{U}:\{1,2, \ldots, d\}^{\mathbb{Z}} \rightarrow \mathbb{R}$.

\section{Equivalence between equilibrium measures and $C^{*}$-Gibbs measures}

First we present two important and well known theorems (see theorems 1.2 and 1.22 in [3] and also [21]).

We will consider without loss of generality that $\beta=1$.

$\mathcal{M}_{\tau}(\Omega)$ denotes the set on invariant probabilities for $\tau$ acting on $\Omega$. 
Theorem 15. (see Theorem 1.2 in [3]) Suppose $U: \Omega \rightarrow \mathbb{R}$ is of Hölder class. Then, there is a unique $\rho \in \mathcal{M}_{\tau}(\Omega)$, for which one can find constants $C_{1}>0, C_{2}>0$, and $P$ such that, for all $s \geq 0$, for all cylinder $\overline{\mid y_{1}^{0}, y_{2}^{0}, \ldots y_{s}^{0}}$ we have

$$
C_{1} \leq \frac{\rho\left(\overline{\mid y_{1}^{0}, y_{2}^{0}, \ldots y_{s}^{0}}\right)}{\exp \left(-P s+\sum_{k=0}^{s-1} U\left(\tau^{k} x\right)\right)} \leq C_{2}
$$

where

$$
x=\left(\ldots x_{-k}, x_{-k+1}, \ldots, x_{-1} \mid x_{1}, \ldots, x_{m}, x_{m+1}, \ldots\right) \in \overline{\mid y_{1}^{0}, y_{2}^{0}, \ldots y_{s}^{0}} \subset \Omega,
$$

We call (25) the Bowen's inequalities.

Definition 16. The probability $\rho=\rho_{U}$ of Theorem 15 is called equilibrium probability for the potential $U$.

Theorem 17. Given $U$ as above and $\rho_{U}$ the equilibrium measure for $U$, then $\rho_{U}$ is the unique probability on $\mathcal{M}_{\tau}(\Omega)$, for which

$$
h\left(\rho_{U}\right)+\int U d \rho_{U}=P(U):=\sup _{\nu \in \mathcal{M}_{\tau}}\left\{h(\nu)+\int U d \nu\right\}
$$

where $h(\nu)$ is the entropy of $\nu$.

For a proof see [16] or [3].

$P(U)$ is called the pressure of $U$. One can show that the $P$ of $(25)$ is equal to such $P(U)$.

Remember that if $\alpha$ is $C^{*}$-Gibbs for $V$, and $V(z, \varphi(z))=\sum_{k=-\infty}^{+\infty} U(z)-$ $U(\varphi(z))$ we also say by abuse of language that $\alpha$ is $C^{*}$-Gibbs for $U: \Omega \rightarrow \mathbb{R}$.

Note that if $\rho$ is an equilibrium probability for a Hölder potential $U$, then, it is also an equilibrium probability for $U+(g \circ \tau)-g+c$, where $c$ is constant and $g: \Omega \rightarrow \mathbb{R}$ is Hölder continuous (see [16]). In this way we can assume without lost of generality that $\rho_{U}$ is an equilibrium probability for a normalized potential $U$. If $U$ is normalized then $P(U)=0$.

If $\alpha$ on $\Omega$ is $C^{*}$-Gibbs for $U$, then, from Remark 2 we have that $\alpha$ is quasi- $C^{*}$-Gibbs for another potential $U$ which is normalized.

Note that given $U$ we are dealing with two definitions: $C^{*}$-Gibbs and Equilibrium. From the above comments we can assume in either case that $U$ is normalized.

The bottom line is: we can assume (see [16]) that the Hölder potential $\tilde{U}=U+(g \circ \tau)-g+c$ is normalized, depends just on future coordinates $\tilde{U}:\{1,2, \ldots, d\}^{\mathbb{N}} \rightarrow \mathbb{R}$ and has pressure zero. 
We will work here (due to Theorem 14 and the above comments) with the case where the probability $\alpha$ - which is $C^{*}$-Gibbs for the potential $U$ - is also a quasi- $C^{*}$-Gibbs probability for the potential $\tilde{U}$ satisfying Pressure $P(\tilde{U})=0$. In this case, if we want to prove expression (25) for such probability $\alpha$ over $\Omega$, this can be simplified just showing that there exist $c_{1}, c_{2}>0$, such that,

$$
c_{1} \leq \frac{\alpha\left(\overline{\left(y_{1}^{0}, y_{2}^{0}, \ldots y_{s}^{0}\right.}\right)}{\exp \left(\sum_{k=0}^{s-1} \tilde{U}\left(\sigma^{k} x\right)\right)} \leq c_{2},
$$

where $\sigma$ is the shift acting on $\{1,2, . ., d\}^{\mathbb{N}}$ and where $x$ is of the form

$$
x=\left(y_{1}^{0}, y_{2}^{0}, \ldots y_{s}^{0}, x_{s+1}, \ldots, x_{m}, x_{m+1}, \ldots\right) \in\{1,2, . ., d\}^{\mathbb{N}} .
$$

Remark 3: Indeed, due to Remark 2 we get that $\tilde{U}=U+(g \circ \tau)-g+c$, where $g$ depends on finite coordinates. Therefore, to show (26) - for $\alpha$ which is $C^{*}$-Gibbs for $U: \Omega \rightarrow \mathbb{R}$ - is equivalent to prove (see details on the proof of Theorem 14) that there exists $C_{1}, C_{2}>0$, such that,

$$
C_{1} \leq \frac{\alpha\left(\overline{\mid y_{1}^{0}, y_{2}^{0}, \ldots y_{s}^{0}}\right)}{\exp \left(\sum_{k=0}^{s-1} U\left(\tau^{k} x\right)\right)} \leq C_{2}
$$

where $\tau$ is the shift acting on $\{1,2, . ., d\}^{\mathbb{Z}}$ and where

$$
x=\left(\ldots x_{-2}, x_{-1} \mid y_{1}^{0}, y_{2}^{0}, \ldots y_{s}^{0}, x_{s+1}, \ldots, x_{m}, x_{m+1}, \ldots\right) \in\{1,2, . ., d\}^{\mathbb{Z}} .
$$

It's important to note that the main equivalence (equilibrium and $C^{*}$ Gibbs) is still valid in a more general setting of a Hölder potential in a general Smale Space. D. Ruelle proved on the setting of hyperbolic diffeomorphisms that Equilibrium implies $C^{*}$-Gibbs in his book [21], see theorems 7.17(b), 7.13(b) and section 7.18). On the other hand Haydn proved in the paper [10] that $C^{*}$-Gibbs implies Equilibrium. Later, the paper [11] presents a shorter proof of the equivalence.

On the two next sections we will present the proof of the following theorem.

Theorem 18. Given a potential $U$ depending on a finite number of coordinates, then, $\alpha$ is the equilibrium measure for $U$, if and only if, $\alpha$ is $C^{*}$-Gibbs for $U$. As the equilibrium probability is unique we get that the $C^{*}$-Gibbs probability for $U$ is unique. 


\section{Equilibrium implies $C^{*}$-Gibbs}

The fact that Equilibrium state implies $C^{*}$-Gibbs was proved by Ruelle in a general setting. The proof is in the book [21] (see theorems 7.17(b), 7.13(b) and section 7.18).

For completeness we will explain the proof on our setting.

We drop the $(x, y)$ on $\varphi_{(x, y)}$ and $\mathcal{O}_{(x, y)}$.

Lemma 19. Let $(\Omega, \tau)$ be the shift on the Bernoulli space $\Omega=\{1,2, \ldots, d\}^{\mathbb{Z}-\{0\}}$ and $\rho_{0}$ be the $\tau$-invariant probability measure which realizes the maximum of the entropy, or, simply the equilibrium state for $U=0$. If $(\mathcal{O}, \varphi)$ is a conjugating homeomorphism, then for any continuous function $f$

$$
\int_{\mathcal{O}} f(\varphi(x)) d \rho_{0}(x)=\int_{\varphi(\mathcal{O})} f(x) d \rho_{0}(x)
$$

Proof: Given

$$
\mathcal{O}=\overline{x_{-n} x_{-n+1} \ldots x_{-1} \mid x_{1} \ldots x_{m-1} x_{m}}
$$

and

$$
\varphi(\mathcal{O})=\overline{y_{-n} y_{-n+1} \ldots y_{-1} \mid y_{1} \ldots y_{m-1} y_{m}},
$$

we have that for any $r>m$ and $k>n$

$$
\begin{gathered}
\rho_{0}\left(\overline{x_{-k} x_{-k+1} \ldots x_{-1} \mid x_{1} \ldots x_{r-1} x_{r}}\right)=d^{-(r+k)}= \\
\rho_{0}\left(\overline{y_{-k} y_{-k+1} \ldots y_{-1} \mid y_{1} \ldots y_{r-1} y_{r}}\right) .
\end{gathered}
$$

We shall prove that equation (28) is valid when $f$ is equal to an characteristic function of an arbitrary cylinder. Note that for this purpose is enough to consider $f$ as the characteristic function of cylinders of the form $\overline{y_{-k} y_{-k+1} \ldots y_{-1} \mid y_{1} \ldots y_{r-1} y_{r}}$. Therefore,

$$
\begin{gathered}
\int_{\mathcal{O}} I \frac{}{y_{-k} y_{-k+1} \ldots y_{-1} \mid y_{1} \ldots y_{r-1} y_{r}}(\varphi(x)) d \rho_{0}(x)= \\
\int_{\varphi(\mathcal{O})} I \frac{y_{-k} y_{-k+1} \ldots y_{-1} \mid y_{1} \ldots y_{r-1} y_{r}}{(y)} d \rho_{0}(y) .
\end{gathered}
$$

From this follows the claim.

The main issue on the above proof is property (29).

We denote by $C^{\alpha}(\Omega)$ the set of $\alpha$ Hölder functions on $\Omega$. 
Lemma 20. (see corollary 7.13 in [21]) Consider the shift space $(\Omega, \tau)$ and $A, B \in C^{\alpha}(\Omega)$. Write for integers $a<0$ and $b>0$

$$
Z_{[a, b]}=\int e^{\sum_{k=a}^{b-1} B \circ \tau^{k}} d \rho_{A}
$$

Then, $Z_{[a, b]}^{-1}\left(\exp \sum_{k=a}^{b-1} B \circ \tau^{k}\right) \rho_{A}$ tends to $\rho_{A+B}$ in the weak star topology, when $a \rightarrow-\infty$ and $b \rightarrow+\infty$.

In particular, taking $A=0$, when $a \rightarrow-\infty$ and $b \rightarrow+\infty$, we get that

$$
Z_{[a, b]}^{-1} e^{\sum_{k=a}^{b-1} B \circ \tau^{k}} \rho_{0} \rightarrow \rho_{B},
$$

where

$$
Z_{[a, b]}=\int e^{\sum_{k=a}^{b-1} B \circ \tau^{k}} d \rho_{0}
$$

Theorem 21. If $\rho_{B}$ is an equilibrium state for a potential $B$ that depends on a finite number of coordinates then it is a $C^{*}$-Gibbs state for $B$.

Proof: The statement holds for $B=0$ by Lemma 19. Moreover, Lemma 20 allow us to extend this result for all $B \in C^{\alpha}\left(\Sigma_{N}\right)$ in the following manner: given $\mathcal{O}$ and the associated $\varphi$

$$
\begin{gathered}
\int_{\varphi(\mathcal{O})} g(x) d \rho_{B}(x)=\lim _{\substack{a \rightarrow-\infty \\
b \rightarrow \infty}} Z_{[a, b]}^{-1} \int_{\varphi(\mathcal{O})} \exp \left(\sum_{k=a}^{b-1} B \circ \tau^{k}(x)\right) g(x) d \rho_{0}(x) \underline{\underline{19}} \\
\lim _{\substack{a \rightarrow-\infty \\
b \rightarrow \infty}} Z_{[a, b]}^{-1} \int_{\mathcal{O}} \exp \left(\sum_{k=a}^{b-1} B \circ \tau^{k} \circ \varphi(x)\right) g \circ \varphi(x) d \rho_{0}(x)= \\
\lim _{\substack{a \rightarrow-\infty \\
b \rightarrow \infty}} Z_{[a, b]}^{-1} \int_{\mathcal{O}} \exp \left(\sum_{k=a}^{b-1} B \circ \tau^{k} \circ \varphi(x)-\sum_{k=0}^{b-1} B \circ \tau^{k}(x)\right) \\
\lim _{\substack{a \rightarrow-\infty \\
b \rightarrow \infty}} Z_{[a, b]}^{-1} \int_{\mathcal{O}} e^{(-V(x, \varphi(x)))} g \circ \varphi(x) \exp \left(\sum_{k=a}^{b-1} B \circ \tau^{k}(x)\right) d \rho_{0}(x)= \\
\int_{\mathcal{O}} e^{(-V(x, \varphi(x)))} g \circ \varphi(x) d \rho_{B}(x) .
\end{gathered}
$$

Since the equality

$$
\int_{\varphi(\mathcal{O})} g(x) d \rho_{B}(x)=\int_{\mathcal{O}} e^{(-V(x, \varphi(x)))} g \circ \varphi(x) d \rho_{B}(x)
$$


was verified for any conjugating homeomorphism $\varphi$ and any $g$, then it follows that $\rho_{B}$ is an $C^{*}$-Gibbs state for $B$.

\section{$7 \quad C^{*}$-Gibbs implies Equilibrium}

Given a $C^{*}$-Gibbs probability $\alpha$ for a potential $U$ that depends on a finite number of coordinates we will show in this section that $\alpha$ is the equilibrium probability for $U$. We shall further assume that the potential $U$ depend only on positive coordinates and is normalized according to the Ruelle operator, i.e.

$$
\sum_{z_{1}, z_{2}, . ., z_{n}=1}^{d} e^{\sum_{j=0}^{n-1} \tilde{U}\left(\sigma^{j}\left(z_{1}, z_{2}, . ., z_{n}, w_{1}, w_{2}, w_{3}, \ldots, w_{m}, \ldots\right)\right.}=1,
$$

for any $w=\left(w_{1}, w_{2}, \ldots, w_{m}, \ldots\right) \in\{1,2, \ldots, d\}^{\mathbb{N}}$ and $n \in \mathbb{N}$. Such assuptions aren't restrictive, since given any potential $W$ that depends on a finite number of coordinates, it's possible to find a function $g$ depending on finite coordinates, and a normalized potential $\tilde{W}$ that depends of future coordinates, such that [16]

$$
W=\tilde{W}+g-g \circ \tau-\lambda
$$

If we show that $\alpha$ is $\tau$-invariant and also satisfies the Bowen's inequalities for $U$, then, it will follow that $\alpha$ is the equilibrium probability for $U$ by Theorem 15.

We will show first that a quasi $C^{*}$-Gibbs probability $\alpha$ for $U$ satisfies the Bowen's inequalities (27) for $U$.

Later we will show that a $C^{*}$-Gibbs probability $\alpha$ is invariant for $\tau$ (see Proposition 26). This will finally show (see Theorem 27) that " $C^{*}$-Gibbs implies Equilibrium".

Note that we want to show (27) but due to Remark 3 we just have to show (26).

We assume $\alpha$ is such that (22) is true, that is, there exists $d_{1}, d_{2}>0$, such that, for any continuous function $g$

$$
\begin{gathered}
d_{1} \int_{\frac{\mid x_{1}^{0}, x_{2}^{0}, \ldots x_{s}^{0}}{}} e^{-V\left(z, \varphi_{(x, y)}(z)\right)} g\left(\varphi_{(x, y)}(z)\right) d \alpha(z) \leq \\
\int_{\frac{y_{1}^{0}, y_{2}^{0}, \ldots y_{s}^{0}}{}} g(z) d \alpha(z) \leq d_{2} \int \frac{}{\mid x_{1}^{0}, x_{2}^{0}, \ldots x_{s}^{0}} e^{-V\left(z, \varphi_{(x, y)}(z)\right)} g\left(\varphi_{(x, y)}(z)\right) d \alpha(z) .
\end{gathered}
$$

We denote $\mathcal{U}=\sup _{x \in \Omega} U(x)-\inf _{x \in \Omega} U(x)$. 
Lemma 22. Given a normalized Hölder potential $U(x)=f\left(x_{1}, x_{2}, . ., x_{r}\right)$, consider $x_{1}^{0} \ldots x_{s}^{0}$ and $y_{1}^{0} \ldots y_{s}^{0}$ fixed, and also $a, b \in\{1,2 \ldots, d\}$ fixed. Let

$$
\begin{aligned}
& x=\left(\ldots x_{-m} x_{-m+1} \ldots x_{-1} \mid x_{1}^{0} \ldots x_{s}^{0} x_{s+1}, x_{s+2}, \ldots x_{m-1} x_{m} \ldots\right) \in \overline{\mid x_{1}^{0}, x_{2}^{0}, \ldots x_{s}^{0}} \\
& y=\left(\ldots x_{-m} x_{-m+1} \ldots x_{-1} \mid y_{1}^{0} \ldots y_{s}^{0} x_{s+1}, x_{s+2}, \ldots x_{m-1} x_{m} \ldots\right) \in \overline{\mid y_{1}^{0}, y_{2}^{0}, \ldots y_{s}^{0}}, \\
& \text { and also } \\
& \quad x_{a}=\left(\ldots x_{-m} x_{-m+1} \ldots x_{-1} \mid x_{1}^{0} \ldots x_{s}^{0} \mathbf{a} x_{s+2} \ldots x_{m-1} x_{m} \ldots\right) \in \overline{\mid x_{1}^{0}, x_{2}^{0}, \ldots x_{s}^{0}} \\
& y_{b}=\left(\ldots x_{-m} x_{-m+1} \ldots x_{-1} \mid y_{1}^{0} \ldots y_{s}^{0} \mathbf{b} x_{s+2} \ldots x_{m-1} x_{m} \ldots\right) \in \overline{\mid y_{1}^{0}, y_{2}^{0}, \ldots y_{s}^{0} .}
\end{aligned}
$$

Assume that $x \sim y$.

Then,

$$
\begin{gathered}
\left|\sum_{k=-\infty}^{\infty} U\left(\tau^{k}\left(x_{a}\right)\right)-U\left(\tau^{k}\left(y_{b}\right)\right)\right| \leq \\
2 r \mathcal{U}+\left|\sum_{k=-\infty}^{\infty} U\left(\tau^{k}(x)\right)-U\left(\tau^{k}(y)\right)\right| .
\end{gathered}
$$

Proof: Let $\mathbb{I}$ the set of indicies for $k$ such that $U\left(\tau^{k}\left(x_{a}\right)\right.$ ) (or, $U\left(\tau^{k}\left(y_{b}\right)\right)$ ) differs from $U\left(\tau^{k}(x)\right)$ (or, $U\left(\tau^{k}(y)\right)$ ). It's easy to see that the cardinality of $\mathbb{I}$ is $r$. Therefore

$$
\begin{gathered}
\left|\sum_{k \in \mathbb{Z}} U\left(\tau^{k}\left(x_{a}\right)\right)-U\left(\tau^{k}\left(y_{b}\right)\right)\right| \leq \\
\left|\sum_{k \in \mathbb{Z} \backslash \mathbb{I}} U\left(\tau^{k}\left(x_{a}\right)\right)-U\left(\tau^{k}\left(y_{b}\right)\right)\right|+\left|\sum_{k \in \mathbb{I}} U\left(\tau^{k}\left(x_{a}\right)\right)-U\left(\tau^{k}\left(y_{b}\right)\right)\right|= \\
\left|\sum_{k \in \mathbb{Z} \backslash \mathbb{I}} U\left(\tau^{k}(x)\right)-U\left(\tau^{k}(y)\right)\right|+\left|\sum_{k \in \mathbb{I}} U\left(\tau^{k}\left(x_{a}\right)\right)-U\left(\tau^{k}\left(y_{b}\right)\right)\right| \leq \\
\left|\sum_{k \in \mathbb{Z} \backslash \mathbb{I}} U\left(\tau^{k}(x)\right)-U\left(\tau^{k}(y)\right)\right|+r \mathcal{U} \leq
\end{gathered}
$$




$$
\left|\sum_{k \in \mathbb{Z}} U\left(\tau^{k}(x)\right)-U\left(\tau^{k}(y)\right)\right|+2 r \mathcal{U}
$$

We will adapt the formulation of Proposition 2.1 in [10] to the present situation.

For fixed $\overline{\mid x_{1}^{0}, x_{2}^{0}, \ldots x_{s}^{0}}$ denote

$$
U_{a}=\overline{\mid x_{1}^{0}, x_{2}^{0}, \ldots, x_{s}^{0}, a}
$$

$a=1,2 . ., d$.

Note that $\sum_{a} \alpha\left(U_{a}\right)=\alpha\left(\overline{\mid x_{1}^{0}, x_{2}^{0}, \ldots x_{s}^{0}}\right)$, in particular

$$
\sum_{a} \alpha\left(U_{a}\right)<d \alpha\left(\overline{\mid x_{1}^{0}, x_{2}^{0}, \ldots x_{s}^{0}}\right) .
$$

Consider now a fixed $\overline{\mid y_{1}^{0}, y_{2}^{0}, \ldots y_{s}^{0}}$ and $\varphi_{a, b}, a=1,2, \ldots, d, b=1,2, \ldots, d$, denotes the conjugating homeomorphism from $U_{a}$ to $\overline{y_{1}^{0}, y_{2}^{0}, \ldots y_{s}^{0} b}=\varphi_{a, b}\left(U_{a}\right)$.

Note also that for each $a$

$$
\alpha\left(\overline{\mid y_{1}^{0}, y_{2}^{0}, \ldots y_{s}^{0}}\right)=\sum_{b=1}^{d} \alpha\left(\varphi_{a, b}\left(U_{a}\right)\right) .
$$

Denote

$$
\begin{gathered}
K=\sup _{m \in \mathbb{N}}\left\{\sum_{k=0}^{m-1}\left[\tilde{U} \tau^{k}(u)-\tilde{U} \tau^{k}(v)\right], \text { where } u, v \in \overline{\mid a_{1}, a_{2}, \ldots, a_{m}},\right. \\
\text { and } \left.\left(a_{1}, a_{2}, \ldots, a_{m}\right) \in\{1,2, . ., d\}^{m}\right\} .
\end{gathered}
$$

On the above expression we ask that $u \sim v$.

Note that if $\alpha$ is $C^{*}$-Gibbs and satisfies (31) we get in particular that

$$
\begin{gathered}
d_{1} \int \frac{}{\mid x_{1}^{0}, x_{2}^{0}, \ldots x_{s}^{0}} e^{-V\left(z, \varphi_{(x, y)}(z)\right)} d \alpha(z) \leq \\
\int \frac{d_{\mid y_{1}^{0}, y_{2}^{0}, \ldots y_{s}^{0}}}{d \alpha} \int \frac{d_{2}}{\mid x_{1}^{0}, x_{2}^{0}, \ldots x_{s}^{0}} e^{-V\left(z, \varphi_{(x, y)}(z)\right)} d \alpha(z) .
\end{gathered}
$$

Proposition 23. Suppose $\alpha$ is quasi-C $C^{*}$ Gibbs for $U$ as above. Then, there exists a constat $c_{1}>0$, such that,

$$
c_{1} \leq e^{-\sum_{k=0}^{s-1} U \tau^{k}(x)} \alpha\left(\overline{\mid x_{1}^{0}, x_{2}^{0}, \ldots x_{s}^{0}}\right)
$$

for any cylinder $\overline{\mid x_{1}^{0}, x_{2}^{0}, \ldots x_{s}^{0}}$ and any $x$ on the cylinder.

The $\alpha$-probability of any cylinder is positive. 
Proof: We assume that (34) is true.

Fix a certain cylinder $\overline{x_{1}^{0}, x_{2}^{0}, \ldots x_{s}^{0}}$ and fix a point $x \in \overline{\mid x_{1}^{0}, x_{2}^{0}, \ldots x_{s}^{0}}$ then choose another cylinder $\overline{\mid y_{1}^{0}, y_{2}^{0}, \ldots y_{s}^{0}}$ with non null probability and a point $y \in \overline{\mid y_{1}^{0}, y_{2}^{0}, \ldots y_{s}^{0}}$. Fix $x \in \overline{\mid x_{1}^{0}, x_{2}^{0}, \ldots x_{s}^{0}}$ and $y \in \overline{\mid y_{1}^{0}, y_{2}^{0}, \ldots y_{s}^{0}}$. Choose $a, b \in$ $\{1,2, \ldots, d\}$ and define $x_{a}$ and $y_{b}$ as

$$
\begin{aligned}
& x_{a}=\left(\ldots x_{-m} x_{-m+1} \ldots x_{-1} \mid x_{1}^{0} \ldots x_{s}^{0} \mathbf{a}, x_{s+2}, \ldots x_{m-1} x_{m} \ldots\right) \in \overline{\mid x_{1}^{0}, x_{2}^{0}, \ldots x_{s}^{0}} \\
& y_{b}=\left(\ldots x_{-m} x_{-m+1} \ldots x_{-1} \mid y_{1}^{0} \ldots y_{s}^{0} \mathbf{b}, x_{s+2}, \ldots x_{m-1} x_{m} \ldots\right) \in \overline{\mid y_{1}^{0}, y_{2}^{0}, \ldots y_{s}^{0}} .
\end{aligned}
$$

we get from Lemma 22 that

$$
\begin{gathered}
\alpha\left(\varphi_{a, b}\left(U_{a}\right)\right) \leq d_{2} \int_{U_{a}} e^{\sum_{k=-\infty}^{\infty} U\left(\tau^{k} \varphi(z)\right)-U\left(\tau^{k}(z)\right)} d \alpha(z) \leq \\
d_{2} \int e^{\sum_{k=0}^{s} U\left(\tau^{k} \varphi(z)\right)-U\left(\tau^{k} y_{b}\right)+U\left(\tau^{k} \varphi(z)\right)-U\left(\tau^{k} x_{a}\right)} e^{\sum_{k=0}^{s} U\left(\tau^{k} y_{b}\right)-U\left(\tau^{k} x_{a}\right)} \\
e^{\sum_{k=s}^{\infty} U\left(\tau^{k} \varphi(z)\right)-U\left(\tau^{k} z\right)} e^{\sum_{k=0}^{\infty} U\left(\tau^{-k} \varphi(z)\right)-U\left(\tau^{-k} z\right)} d \alpha(z) \leq \\
d_{2} e^{2 K+r \mathcal{U}} e^{\sum_{k=0}^{s-1}\left[\tilde{U} \tau^{k}\left(y_{b}\right)-\tilde{U} \tau^{k}\left(x_{a}\right)\right]} \alpha\left(U_{a}\right) \leq \\
d_{2} e^{2 K+3 r \mathcal{U}} e^{\sum_{k=0}^{s-1}\left[\tilde{U} \tau^{k}(y)-\tilde{U} \tau^{k}(x)\right]} \alpha\left(U_{a}\right) .
\end{gathered}
$$

Then, from (33)

$\alpha\left(\overline{\left(y_{1}^{0}, y_{2}^{0}, \ldots y_{s}^{0}\right.}\right)=\sum_{b=1}^{d} \alpha\left(\varphi_{a, b}\left(U_{a}\right)\right) \leq d_{2} d e^{2 K+3 r \mathcal{U}} e^{\sum_{k=0}^{s-1}\left[U \tau^{k}(y)-U \tau^{k}(x)\right]} \alpha\left(U_{a}\right)$.

From this and from (30) we get

$$
1=\sum_{y_{1}^{0}, y_{2}^{0}, \ldots y_{s}^{0}=1}^{d} \alpha\left(\overline{\mid y_{1}^{0}, y_{2}^{0}, \ldots y_{s}^{0}}\right) \leq d_{2} d e^{2 K+3 r \mathcal{U}} e^{-\sum_{k=0}^{s-1} U \tau^{k}(x)} \alpha\left(U_{a}\right),
$$

and, finally, for $x=\left(\ldots, x_{-t}, \ldots, x_{-2}, x_{-1} \mid x_{1}, x_{2}, . ., x_{t}, \ldots\right) \in \overline{\mid x_{1}^{0}, x_{2}^{0}, \ldots x_{s}^{0}}$

$$
d=\sum_{a=1}^{d} \sum_{y_{1}^{0}, y_{2}^{0}, \ldots y_{s}^{0}=1}^{d} \alpha\left(\overline{\mid y_{1}^{0}, y_{2}^{0}, \ldots y_{s}^{0}}\right) \leq \sum_{a=1}^{d} d_{2} d e^{2 K+3 r \mathcal{U}} e^{-\sum_{k=0}^{s-1} U \tau^{k}(x)} \alpha\left(U_{a}\right)=
$$




$$
d_{2} d e^{2 K+3 r \mathcal{U}} e^{-\sum_{k=0}^{s-1} U \tau^{k}(x)} \alpha\left(\overline{\mid x_{1}^{0}, x_{2}^{0}, \ldots x_{s}^{0}}\right) .
$$

This also shows that the $\alpha$-probability of any cylinder $\overline{\mid x_{1}^{0}, x_{2}^{0}, \ldots x_{s}^{0}}$ is positive when $\alpha$ is quasi- $C^{*}$-Gibbs.

By Proposition 13 we get that any cylinder of the form $\overline{x_{-m} \ldots x_{-1} \mid x_{1} x_{2} \ldots x_{s}}$ has positive $\alpha$-probability.

Proposition 24. There exists a constant $c_{2}>0$, such that,

$$
e^{-\sum_{k=0}^{s-1} U \tau^{k}(x)} \alpha\left(\overline{\mid x_{1}^{0}, x_{2}^{0}, \ldots x_{s}^{0}}\right) \leq c_{2},
$$

for any cylinder $\overline{\mid x_{1}^{0}, x_{2}^{0}, \ldots x_{s}^{0}}$ and any $x$ on the cylinder.

The $\alpha$-probability of any cylinder is positive.

Proof: We assume that (34) is true.

Again consider fixed $x \in \overline{\mid x_{1}^{0}, x_{2}^{0}, \ldots x_{s}^{0}}$ and $y \in \overline{\mid y_{1}^{0}, y_{2}^{0}, \ldots y_{s}^{0}}$. Choose $a, b \in$ $\{1,2, \ldots, d\}$ and define $x_{a}$ and $y_{b}$ as

$$
\begin{aligned}
& x_{a}=\left(\ldots x_{-m} x_{-m+1} \ldots x_{-1} \mid x_{1}^{0} \ldots x_{s}^{0} \mathbf{a}, x_{s+2}, \ldots x_{m-1} x_{m} \ldots\right) \in \overline{\mid x_{1}^{0}, x_{2}^{0}, \ldots x_{s}^{0}} \\
& y_{b}=\left(\ldots x_{-m} x_{-m+1} \ldots x_{-1} \mid y_{1}^{0} \ldots y_{s}^{0} \mathbf{b}, x_{s+2}, \ldots x_{m-1} x_{m} \ldots\right) \in \overline{\mid y_{1}^{0}, y_{2}^{0}, \ldots y_{s}^{0}} .
\end{aligned}
$$

Using an analogous reasoning as in proposition 24. But now we use the function $g(z)=e^{V(z, \varphi(z))}$ in the first inequality of (31). After some algebraic work similar to the former demonstration we reach

$$
\begin{gathered}
\alpha\left(U_{a}\right) \leq \frac{1}{d_{1}} e^{2 K+r \mathcal{U}} e^{\sum_{k=0}^{s-1}\left[U \tau^{k}\left(x_{a}\right)-U \tau^{k}\left(y_{b}\right)\right]} \alpha\left(\varphi_{a, b}\left(U_{a}\right)\right) \leq \\
\frac{1}{d_{1}} e^{2 K+3 r \mathcal{U}} e^{\sum_{k=0}^{s-1}\left[U \tau^{k}(x)-U \tau^{k}(y)\right]} \alpha\left(\varphi_{a, b}\left(U_{a}\right)\right) .
\end{gathered}
$$

Therefore,

$$
\begin{gathered}
e^{\sum_{k=0}^{s-1} U \tau^{k}(y)} \alpha\left(\overline{\mid x_{1}^{0}, x_{2}^{0}, \ldots x_{s}^{0}}\right)=e^{\left.\sum_{k=0}^{s-1} U \tau^{k}(y)\right]} \sum_{a=1}^{d} \alpha\left(U_{a}\right) \leq \\
\frac{1}{d_{1}} e^{2 K+3 r \mathcal{U}} e^{\sum_{k=0}^{s-1} U \tau^{k}(x)} \sum_{a=1}^{d} \alpha\left(\varphi_{a, b}\left(U_{a}\right)\right) .
\end{gathered}
$$


Finally, as $\overline{y_{1}^{0}, y_{2}^{0}, \ldots y_{s}^{0} b}=\varphi_{a, b}\left(U_{a}\right)$ we get from (30) and (35)

$$
\begin{gathered}
d \alpha\left(\overline{\mid x_{1}^{0}, x_{2}^{0}, \ldots x_{s}^{0}}\right)=\sum_{b=1}^{d} \sum_{y_{1}^{0}, y_{2}^{0}, \ldots y_{s}^{0}=1}^{d} e^{\sum_{k=0}^{s-1} U \tau^{k}(y)} \alpha\left(\overline{\mid x_{1}^{0}, x_{2}^{0}, \ldots x_{s}^{0}}\right) \leq \\
\frac{e^{2 K+3 r \mathcal{U}}}{d_{1}} e^{\sum_{k=0}^{s-1} U \tau^{k}(x)} \sum_{a=1}^{d} \sum_{b=1}^{d} \sum_{y_{1}^{0}, y_{2}^{0}, \ldots y_{s}^{0}=1}^{d} \alpha\left(\varphi_{a, b}\left(U_{a}\right)\right)=\frac{d e^{2 K+3 r \mathcal{U}}}{d_{1}} e^{\sum_{k=0}^{s-1} U \tau^{k}(x)} .
\end{gathered}
$$

This shows the claim of the proposition.

Now we have to show that $\alpha$ is invariant by $\tau$.

Corollary 25. If $\alpha_{1}$ and $\alpha_{2}$ are quasi $C^{*}$-Gibbs for $U$, where

$$
U\left(\ldots, x_{-n}, \ldots, x_{-2}, x_{-1} \mid x_{1}, x_{2}, \ldots, x_{r}, x_{r+1}, \ldots x_{m} \ldots\right)=f\left(x_{1}, x_{2}, \ldots, x_{r}\right)
$$

for some fixed $r$ and function $f:\{1,2, \ldots, d\}^{r} \rightarrow \mathbb{R}$, then $\alpha_{1}$ is absolutely continuous with respect to $\alpha_{2}$.

Proof: We assume that $U$ is normalized. Suppose $\alpha_{1}$ and $\alpha_{2}$ are quasi $C^{*}$-Gibbs for $U$.

Expression (26) for $\alpha_{1}$ and $\alpha_{2}$ will determine, respectively, constants $d_{1}^{1}, d_{2}^{1}$ and $d_{1}^{2}, d_{2}^{2}$.

From last Propositions there exist constants $Y_{1}>0$ and $Y_{2}>0$, such that, for any cylinder $\overline{\mid x_{1}, x_{2}, . ., x_{n}}$ and for any point $x$ in this cylinder we get

$$
\frac{\alpha_{1}\left(\overline{\left(x_{1}, x_{2}, . ., x_{n}\right.}\right)}{e^{\sum_{k=0}^{n-1} U\left(\tau^{k}(x)\right)}} \leq Y_{1}
$$

and

$$
Y_{2} \leq \frac{\alpha_{2}\left(\overline{\mid x_{1}, x_{2}, . ., x_{n}}\right)}{e^{\sum_{k=0}^{n-1} U\left(\tau^{k}(x)\right)}}
$$

Therefore,

$$
\frac{Y_{2}}{Y_{1}} \alpha_{1}\left(\overline{\mid x_{1}, x_{2}, . ., x_{n}}\right) \leq \alpha_{2}\left(\overline{\mid x_{1}, x_{2}, . ., x_{n}}\right) .
$$

Now consider a cylinder set of the form

$$
\left(\overline{x_{-m}, \ldots x_{-1} \mid x_{1}, x_{2}, . ., x_{n}}\right) .
$$

Expression (23) for $\alpha_{1}$ and $\alpha_{2}$ will determine, respectively, constants $b_{1}^{1}, b_{2}^{1}$ and $b_{1}^{2}, b_{2}^{2}$.

Then, by Proposition 13 we get that 


$$
\begin{gathered}
b_{1}^{1} \alpha_{1}\left(\overline{x_{-m}, \ldots x_{-1} \mid x_{1}, x_{2}, . ., x_{n}}\right) \leq \alpha_{1}\left(\overline{\mid x_{-m}, \ldots x_{-1} x_{1}, x_{2}, . ., x_{n}}\right) \leq \\
\frac{Y_{1}}{Y_{2}} \alpha_{2}\left(\overline{\mid x_{-m}, \ldots x_{-1} x_{1}, x_{2}, . ., x_{n}}\right) \leq \frac{Y_{1}}{Y_{2}} b_{2}^{2} \alpha_{2}\left(\overline{x_{-m}, \ldots x_{-1} \mid x_{1}, x_{2}, . ., x_{n}}\right) .
\end{gathered}
$$

The Borel sigma-algebra over $\Omega$ is generated by the set of cylinders of the form $\overline{x_{-m}, \ldots x_{-1} \mid x_{1}, x_{2}, . ., x_{n}}$.

As the probability $\alpha_{j}(B), j=1,2$, of a Borel set $B$ is obtained, respectively, as an exterior probability using probabilities of the generators we finally get that the analogous inequalities as in (36) are true with the same same constants, that is,

$$
b_{1}^{1} \alpha_{1}(B) \leq \frac{Y_{1}}{Y_{2}} b_{2}^{2} \alpha_{2}(B) .
$$

Therefore, $\alpha_{1}$ is absolutely continuous with respect to $\alpha_{2}$.

Proposition 26. Assume $\alpha$ is $C^{*}$-Gibbs for $U$, then, $\alpha$ is invariant for $\tau$.

Proof: From Corollary 25 we get that any two $C^{*}$-Gibbs probabilities for $U$ are absolutely continuous with respect to each other.

Suppose $\alpha$ is $C^{*}$-Gibbs, then, $\alpha_{1}=\tau^{*}(\alpha)$ is also $C^{*}$-Gibbs by Proposition 10. If $\alpha \neq \tau^{*}(\alpha)$ then, following Theorem 2.5 in [11] we get that $\rho_{1}=$ $\left|\alpha_{1}-\alpha\right|+\alpha_{1}-\alpha$ and $\rho_{2}=\left|\alpha_{1}-\alpha\right|-\alpha_{1}+\alpha$ are also $C^{*}$-Gibbs. But $\rho_{1}$ and $\rho_{2}$ are singular with respect to each other and this is a contradiction.

Therefore, $\alpha=\tau^{*}(\alpha)$.

Theorem 27. Suppose $U: \Omega \rightarrow \mathbb{R}$ is of the form

$$
U\left(\ldots, x_{-n}, \ldots, x_{-2}, x_{-1} \mid x_{1}, x_{2}, \ldots, x_{r}, x_{r+1}, \ldots x_{m} \ldots\right)=f\left(x_{1}, x_{2}, \ldots, x_{r}\right),
$$

for some fixed $r$ and fixed function $f:\{1,2, \ldots, d\}^{r} \rightarrow \mathbb{R}$.

If $\alpha$ is $C^{*}$-Gibbs for the potential $U$ then $\alpha$ is the equilibrium state for $U$.

Proof: As we know by Proposition 26 that $\alpha$ is $\tau$ invariant and, moreover, we also know that $\alpha$ is quasi- $C^{*}$ invariant for another normalized potential, it follows from Proposition 23, Proposition 24 and Theorem 15 that $\alpha$ is the equilibrium probability for $U$

Another conclusion one can get from the above reasoning is that for potentials that depends on finite coordinates the concepts of quasi $C^{*}$-Gibbs and $C^{*}$-Gibbs are equivalent on the lattice $\mathbb{Z}$. 


\section{Construction of the $C^{*}$-Algebra}

Remember that we consider the groupoid $G \subset \Omega \times \Omega$ of all pair of points which are related by the homoclinic equivalence relation.

Remember also that we consider on $G$ the topology generated by sets of the form

$$
\left\{\left(z, \varphi_{(x, y)}(z)\right) \mid \text { where } z \in \mathcal{O}_{(x, y)} \text { and } x, y \in \Omega \text { such that } x \sim y\right\} .
$$

This topology is Hausdorff [20].

We denote by $[x]$ the class of $x \in \Omega$. For each $x$ the set of elements on the class $[x]$ is countable.

We now come to the construction of the noncommutative algebra. Let $\mathcal{C}_{c}(G)$ be the linear space of complex continuous functions with compact support on $G$. If $A, B \in \mathcal{C}_{c}(G)$ we define the product $A * B$ by

$$
(A * B)(x, y)=\sum_{z \in[x]} A(x, z) B(z, y) .
$$

Note that if $(x, y) \in G$ then they are conjugated and so the sum is over all $z$ that are conjugated to $x$ and $y$.

Note that there are only finitely many nonzero terms in the above sum because the functions $A, B$ have compact support [20].

Considering the above, $A * B \in \mathcal{C}_{c}(G)$ as one checks readily, so that $\mathcal{C}_{c}(G)$ becomes an associative complex algebra. An involution $A \rightarrow A^{*}$ is defined by

$$
A^{*}(x, y)=\overline{A(y, x)}
$$

where the bar denotes complex conjugation.

For each equivalence class $[x]$ of conjugated points of $\Omega$ there is a representation $\pi_{[x]} \rightarrow \mathbb{C}$ in the Hilbert space $l^{2}([x])$ of square summable functions $[x] \rightarrow \mathbb{C}$, such that

$$
\left(\left(\pi_{[x]} A\right) \xi(y)=\sum_{z \in[x]} A(y, z) \xi(z)\right.
$$

for $\xi \in l^{2}([x])$. Denoting by $\left\|\pi_{[x]} A\right\|$ the operator norm, we write

$$
\|A\|=\sup _{[x]}\left\|\pi_{[x]} A\right\| .
$$

$I_{D}$ (the indicator function of the diagonal $D$ ) is such that for any $A \in$ $\mathcal{C}_{c}(G)$ we get $I_{D} * A=A * I_{D}=A$.

The completion of $\mathcal{C}_{c}(G)$ with respect to this norm is separable. It is called the reduced $C^{*}$-algebra which is denoted by $C_{r}^{*}(G)$. The unity element $I_{D}$ is contained in this $C^{*}$ algebra. 
Remark 28. If $A \in \mathcal{C}_{c}(G)$ and $t \in \mathbb{R}$, we write

$$
\left(\sigma^{t} A\right)(x, y)=e^{i V(x, y) t} A(x, y)
$$

defining a one-parameter group $\left(\sigma^{t}\right)$ of *-automorphisms of $\mathcal{C}_{c}(G)$ and a unique extension to a one parameter group of *-automorphisms of $C_{r}^{*}(G)$.

We say that $A \in \mathcal{C}_{c}(G)$ is analytic (a classical terminology on $C^{*}$-algebras) if the real variable $t$ on the function $t \rightarrow \sigma^{t} A$ can be extended to the complex variable $z \in \mathbb{C}$. Under our assumptions this will be always the case. Therefore, $\sigma^{-\beta i} A$ is well defined.

Definition 29. A state $\omega$ on $C_{r}^{*}(G)$ is a linear functional $\omega: C_{r}^{*}(G) \rightarrow \mathbb{C}$, such that, $\omega\left(A * A^{*}\right) \geq 0$, and $\omega\left(I_{D}\right)=1$ (see [4]).

Such state $\omega$ is sometimes called a dynamical $C^{*}$-state.

Definition 30. A state $\omega$ is invariant if $\omega \circ \sigma^{t}=\omega$, for all $t \in \mathbb{R}$.

It is of paramount importance to be able to substitute the above real value $t$ by the complex number $\beta i$ (where $\beta$ is real). We refer the reader to Propositions 5.3.6 e 5.3.7 in [4] for the technical details of this claim.

Definition 31. Given a modular function $V: G \rightarrow \mathbb{R}$ and the associated $\sigma_{t}, t \in \mathbb{R}$, we say that an invariant state $\omega: C_{r}^{*}(G) \rightarrow \mathbb{C}$ satisfies the $\boldsymbol{K M} \boldsymbol{S}$ boundary condition for $V$ and $\beta \in \mathbb{R}$, if for all $A, B \in C_{r}^{*}(G)$, there is a continuous function $F$ on $\{z \in \mathbb{C}: 0 \leq \operatorname{Im}(z) \leq \beta\}$, holomorphic in $\{z \in \mathbb{C}: 0<\operatorname{Im}(z)<\beta\}$, and such that for any real $t$

$$
\omega\left(\sigma^{t} A * B\right)=F(t), \quad \omega\left(B * \sigma^{t} A\right)=F(t+i \beta)
$$

Note that using (40) we have that $F(0)=\omega(A \cdot B)$ and

$$
F(0)=F(-\beta i+\beta i)=\omega\left(B * \sigma^{-\beta i} A\right) .
$$

Therefore, for any $A, B$ we get

$$
\omega(A * B)=\omega\left(B * e^{-i \beta} A\right)
$$

which is the classical KMS condition for $\omega$ according to [4] (see Propositions 5.3.6 e 5.3.7 there). This condition is equivalent to KMS boundary condition. 
Theorem 32. If $\mu$ is a probability measure on $\Omega$ then a state $w=\hat{\mu}$ on $C_{r}^{*}(G)$ can be defined for any $A \in \mathcal{C}_{c}(G)$ by

$$
\hat{\mu}(A)=\int A(x, x) d \mu(x)
$$

\section{Proof:}

$\hat{\mu}$ is bounded with respect to the above defined norm.

First note that it's easy to verify that $\hat{\mu}$ is linear, and for any $A$ we have $\hat{\mu}\left(A * A^{*}\right) \geq 0$ and moreover $\hat{\mu}\left(I_{D}\right)=1$. Now, note that since the diagonal $D$ is a compact set, then any continuous function $A: G \rightarrow \mathbb{C}$ has a maximum at $D$, therefore (41) is well defined for continuous function. $\hat{\mu}$ is also well defined on the $C^{*}$-algebra.

Definition 33. A probability $\nu$ on $\Omega$ is called a KMS probability for the modular function $V$ if the state $\hat{\nu}$ on $C_{r}^{*}(G)$ defined by

$$
\hat{\nu}(A)=\int A(x, x) \nu(d x)
$$

satisfies the KMS condition for $V$. Here $G$ is the groupoid given by the homoclinic equivalence relation.

This probability is sometimes called quasi-stationary (see [5]).

The next claim was proved on [20]. For completeness we will present a proof of this claim with full details.

Theorem 34. If the probability $\alpha$ on $\Omega$ is a $C^{*}$-Gibbs probability with respect to $V$ and $\beta$, then, $\hat{\alpha}$ is a KMS probability for the modular function $\beta V$. The associated $\hat{\alpha}$ is a $C^{*}$ dynamical state for the $C_{r}^{*}(G)$ algebra given by the groupoid obtained by the homoclinic equivalence relation and satisfies the KMS boundary condition.

Proof: Suppose $\alpha$ is a $C^{*}$-Gibbs state with respect to $\beta V$. We assume $\beta=1$.

$\hat{\alpha}$ is $\sigma^{t}$ invariant if for all $t \in \mathbb{C}$ it's true that

$$
\int \sigma^{t} A(x, x) \alpha(d x)=\int A(x, x) \alpha(d x)
$$

which by definition (39) it's equivalent to

$$
\int e^{i V(x, x) t} A(x, x) \alpha(d x)=\int A(x, x) \alpha(d x)
$$


but since $V(x, x)=0$ then the state have to be $\sigma^{t}$ invariant.

Now we will show that if $A, B \in \mathcal{C}_{c}(G)$, then

$$
\hat{\alpha}\left(\sigma^{t} A * B\right)=\int \alpha(d x) \sum_{y \in[x]} e^{i V(x, y) t} A(x, y) \cdot B(y, x)
$$

extends to an entire function (just change $t$ to $z \in \mathbb{C}$ ). For this purpose we will pick $t_{0} \in \mathbb{C}$ and show that

$$
\lim _{t \rightarrow t_{0}} \frac{\hat{\alpha}\left(\sigma^{t} A * B\right)-\hat{\alpha}\left(\sigma^{t_{o}} A * B\right)}{t-t_{0}}
$$

exist. Indeed, the limit (43) is equivalent to

$$
\begin{gathered}
\lim _{t \rightarrow t_{0}} \frac{1}{t-t_{0}}\left(\int \alpha(d x) \sum_{y \in[x]} e^{i V(x, y) t} A(x, y) \cdot B(y, x)-\right. \\
\left.\int \alpha(d s) \sum_{y \in[s]} e^{i V(s, y) t_{0}} A(s, y) \cdot B(y, s)\right)= \\
\lim _{t \rightarrow t_{0}}\left(\int \alpha(d x) \sum_{y \in[x]} \frac{\left(e^{i V(x, y) t}-e^{i V(x, y) t_{0}}\right)}{t-t_{0}} A(x, y) \cdot B(y, x)\right) .
\end{gathered}
$$

Always have in mind that for each $x$ the summation is over finite terms.

Let $R$ be a closed ball of radius 1 centered in $t_{0}$. So we can consider the continuous function $f_{t_{0}}: R \backslash\left\{t_{0}\right\} \times \operatorname{supp}(A) \rightarrow \mathbb{C}$

$$
f_{t_{0}}(t, x)=\sum_{y \in[x]} \frac{\left(e^{i V(x, y) t}-e^{i V(x, y) t_{0}}\right)}{t-t_{0}} A(x, y) \cdot B(y, x)
$$

To extend $f_{t_{0}}$ for the case $t=t_{0}$ we need to solve the limit

$$
\begin{gathered}
L_{t_{0}}(x)=\lim _{t \rightarrow t_{0}} \sum_{y \in[x]} \frac{\left(e^{i V(x, y) t}-e^{i V(x, y) t_{0}}\right)}{t-t_{0}} A(x, y) \cdot B(y, x)= \\
\sum_{y \in[x]} \lim _{t \rightarrow t_{0}} \frac{\left(e^{i V(x, y) t}-e^{i V(x, y) t_{0}}\right)}{t-t_{0}} A(x, y) \cdot B(y, x)= \\
\sum_{y \in[x]} i V(x, y) e^{i V(x, y) t_{0}} A(x, y) \cdot B(y, x) .
\end{gathered}
$$


So define $f_{t_{0}}\left(t_{0}, x\right)=L_{t_{0}}(x)$.

In this way $f_{t_{0}}$ is a continuous function defined on a compact domain. Therefore we may assume that both it's real and imaginary parts are limited by a value $M$ in the domain. Consider a sequence of functions indexed by the $t$ variable, $\left\{f_{t_{0}}\left(t_{n}, x\right)\right\}_{n \in \mathbb{N}^{*}}$ that converge to $L_{t_{0}}(x)$ when $n \rightarrow \infty$, e.g. $f_{t_{0}}\left(t_{0}+(1+i) / n, x\right)$. In this way the dominated convergence theorem assures that the limit (43) is equal to the integral:

$$
\int \alpha(d x) L_{t_{0}}(x)
$$

Indeed formally what we have is,

$$
\begin{gathered}
\int \alpha(d x) L_{t_{0}}(x)=\int \alpha(d x) \sum_{y \in[x]} i V(x, y) e^{i V(x, y) t_{0}} A(x, y) \cdot B(y, x)= \\
\int \alpha(d x) \lim _{n \rightarrow \infty} \sum_{y \in[x]} \frac{\left(e^{i V(x, y) t_{n}}-e^{i V(x, y) t_{0}}\right)}{t_{n}-t_{0}} A(x, y) \cdot B(y, x)= \\
\lim _{n \rightarrow \infty} \int \alpha(d x) \sum_{y \in[x]} \frac{\left(e^{i V(x, y) t_{n}}-e^{i V(x, y) t_{0}}\right)}{t_{n}-t_{0}} A(x, y) \cdot B(y, x)= \\
\lim _{n \rightarrow \infty} \frac{\hat{\alpha}\left(\sigma^{t_{n}} A * B\right)-\hat{\alpha}\left(\sigma^{t_{o}} A * B\right)}{t_{n}-t_{0}}
\end{gathered}
$$

Now since the sequence was arbitrary we could remake these calculations to any desired convergent sequence with the same result, therefore (46) is equal to

$$
\lim _{t \rightarrow t_{0}} \frac{\hat{\alpha}\left(\sigma^{t} A * B\right)-\hat{\alpha}\left(\sigma^{t_{o}} A * B\right)}{t-t_{0}},
$$

what proves existence of the limit in equation (43). This allow us to conclude that $\hat{\alpha}\left(\sigma^{t} A * B\right)$ is an holomorphic function everywhere.

Let $F(t)=\hat{\alpha}\left(\sigma^{t} A * B\right)$. Using a partition of unity on supp $A$ we may write $A=\sum A_{j}$, where supp $A_{j} \subset W_{j}=\left\{\left(z, \varphi_{j}(z)\right): z \in \mathcal{O}_{j}\right\}$, and $\left(\mathcal{O}_{j}, \varphi_{j}\right)$ is a conjugating homeomorphism. Since supp $A$ is a compact set then we may assume the summation to occur over a finite amount of elements. Thus

$$
\begin{gathered}
F(t)=\int_{\Omega} \sum_{j} \alpha(d x) A_{j}\left(x, \varphi_{j} x\right) B\left(\varphi_{j} x, x\right) \exp \left(i V\left(x, \varphi_{j} x\right) t\right)= \\
\sum_{j} \int_{\mathcal{O}_{j}} \alpha(d x) A_{j}\left(x, \varphi_{j} x\right) B\left(\varphi_{j} x, x\right) \exp \left(i V\left(x, \varphi_{j} x\right) t\right)
\end{gathered}
$$


and therefore

$$
F(t+i)=\sum_{j} \int_{\mathcal{O}_{j}}\left[e^{-V\left(x, \varphi_{j} x\right)} \alpha(d x)\right] A_{j}\left(x, \varphi_{j} x\right) B\left(\varphi_{j} x, x\right) \exp \left(i V\left(x, \varphi_{j} x\right) t\right)
$$

If $\alpha$ is an $C^{*}$-Gibbs state by (15) we have that

$$
\begin{gathered}
F(t+\beta i)=\sum_{j} \int_{\varphi_{j}\left(\mathcal{O}_{j}\right)} \alpha(d y) B\left(y, \varphi_{j}^{-1} y\right) A_{j}\left(\varphi_{j}^{-1} y, y\right) \exp \left(i V\left(\varphi_{j}^{-1} y, y\right) t\right)= \\
\sum_{j} \int_{\varphi_{j}\left(\mathcal{O}_{j}\right)} \alpha(d y) B\left(y, \varphi_{j}^{-1} y\right) \sigma^{t} A_{j}\left(\varphi_{j}^{-1} y, y\right)= \\
\int_{\Omega} \sum_{j} \alpha(d y) B\left(y, \varphi_{j}^{-1} y\right) \sigma^{t} A_{j}\left(\varphi_{j}^{-1} y, y\right)= \\
\int_{\Omega} \alpha(d y)\left(B * \sigma^{t} A\right)(y, y)=\hat{\alpha}\left(B * \sigma_{t} A\right)
\end{gathered}
$$

so that $\hat{\alpha}$ satisfies the KMS condition.

\section{References}

[1] A. Baravieira, R. Leplaideur and A. O. Lopes, Ergodic optimization, zero temperature limits and the max-plus algebra, XXIX Coloq. Bras. Mat - IMPA - Rio de Janeiro, 2013.

[2] R. Bissacot and B. Kimura, Gibbs Measures on Multidimensional Subshifts, preprint, 2016.

[3] R. Bowen, Equilibrium States and the Ergodic Theory of Anosov Dieomorphisms, Springer, 1975.

[4] O. Bratteli and D. Robinson, Operator Algebras and Quantum Statistical Mechanics I, Springer Verlag.

[5] G. Castro, A. O. Lopes and G. Mantovani, Haar systems, KMS states on von Neumann algebras and $C^{*}$-algebras on dynamically defined groupoids and Noncommutative Integration, preprint 2017

[6] L. Cioletti and A. O. Lopes, Interactions, Specifications, DLR probabilities and the Ruelle Operator in the One-Dimensional Lattice, Discrete and Cont. Dyn. Syst. - Series A, Vol 37, Number 12, 6139 - 6152 (2017) 
[7] G. DellAntonio, Lectures on the Mathematics of Quantum Mechanics II, Atlantis Press, 2016.

[8] R. Exel and A. O. Lopes, $C^{*}$-Algebras, approximately proper equivalence relations and Thermodynamic Formalism, Erg Theo and Dyn Syst, Vol 24, pp 1051-1082 (2004).

[9] R. Exel and A. O. Lopes, C*- Algebras and Thermodynamic Formalism, Sao Paulo Journal of Mathematical Sciences 2, 1 (2008), 285-307

[10] N. T. A. Haydn, On Gibbs and equilibrium states, Ergod. Th. and Dynam. Sys., (7): 119-132, 1987.

[11] N. T. A. Haydn and D. Ruelle, Equivalence of Gibbs and Equilibrium states for homeomorphisms satisfying expansiveness and specification. Comm. in Math. Phys., 148, 155-167, 1992

[12] A. Kumjian and J. Renault, KMS states on $C^{*}$-Algebras associated to expansive maps, Proc. AMS Vol. 134, No. 7, 2067-2078, 2006,

[13] A. O. Lopes and E. Oliveira, Continuous groupoids on the symbolic space, quasi-invariant probabilities for Haar systems and the HaarRuelle operator, to appear in Bull of the Braz. Math. Soc. (on line 2018)

[14] I. Putnam, $C^{*}$-algebras from Smale spaces. Canad. J. Math. 48, no. 1, 175-195, 1996.

[15] I. Putnam and J. Spielberg, The Structure of $C^{*}$-Algebras associated with hyperbolic dynamical systems, J. Funct. Anal. 163, no. 2, 279-299. 1999.

[16] W. Parry and M. Pollicott, Zeta Functions and the Periodic Orbit Structure of Hyperbolic Dynamics. 1990.

[17] G. K. Pedersen, $C^{*}$ algebras and Their automorphism groups. Academic Press, 1979.

[18] J. Renault, A Groupoid approach to $C^{*}$-algebras, Lecture Notes in Mathematics 793 Springer-Verlag, 1980.

[19] J. Renault, $C^{*}$-Algebras and Dynamical Systems, XXVII Coloquio Bras. de Matematica - IMPA - Rio de Janeiro, 2009 
[20] D. Ruelle, Noncommutative algebras for hyperbolic diffeomorphisms. Inventiones mathematicae, (93):1-13, 1988.

[21] D. Ruelle, Thermodynamic Formalism. Cambridge University Press, 2 edition, 2004.

[22] C. K. Thomsen, $C^{*}$-algebras of homoclinic and heteroclinic structure in expansive dynamics, Memoirs of Amer. Math. Soc. 206, No 970, 2010 